\title{
Distinct Adipogenic and Fibrogenic Differentiation Capacities of Mesenchymal Stromal Cells from Pancreas and White Adipose Tissue
}

\author{
Heja Aga 1,2 (D), George Soultoukis ${ }^{2,3}$, Mandy Stadion ${ }^{1,2}$, Francisco Garcia-Carrizo ${ }^{3}$ (D), Markus Jähnert ${ }^{1,2}$ (D), \\ Pascal Gottmann ${ }^{1,2}$ (D), Heike Vogel ${ }^{1,2,4,5}$, Tim J. Schulz ${ }^{2,3,6}$ and Annette Schürmann 1,2,6,*(D)
}

check for updates

Citation: Aga, H.; Soultoukis, G.; Stadion, M.; Garcia-Carrizo, F.; Jähnert, M.; Gottmann, P.; Vogel, H.; Schulz, T.J.; Schürmann, A. Distinct Adipogenic and Fibrogenic Differentiation Capacities of Mesenchymal Stromal Cells from Pancreas and White Adipose Tissue. Int. J. Mol. Sci. 2022, 23, 2108. https://doi.org/10.3390/ ijms23042108

Academic Editor:

Hideyuki Yamawaki

Received: 25 January 2022

Accepted: 9 February 2022

Published: 14 February 2022

Publisher's Note: MDPI stays neutral with regard to jurisdictional claims in published maps and institutional affiliations.

Copyright: (C) 2022 by the authors. Licensee MDPI, Basel, Switzerland. This article is an open access article distributed under the terms and conditions of the Creative Commons Attribution (CC BY) license (https:// creativecommons.org/licenses/by/ $4.0 /)$.
1 Department of Experimental Diabetology, German Institute of Human Nutrition Potsdam-Rehbruecke (DIfE), 14558 Nuthetal, Germany; heja.aga@dife.de (H.A.); mandy.stadion@gmx.de (M.S.); markus.jaehnert@dife.de (M.J.); pascal.gottmann@dife.de (P.G.); heikevogel@dife.de (H.V.)

2 German Center for Diabetes Research (DZD), München-Neuherberg, 85764 München, Germany; george.soultoukis@dife.de (G.S.); tim.schulz@dife.de (T.J.S.)

3 Department of Adipocyte Development and Nutrition, German Institute of Human Nutrition Potsdam-Rehbruecke (DIfE), 14558 Nuthetal, Germany; francisco.garcia@dife.de

4 Research Group Genetics of Obesity, German Institute of Human Nutrition Potsdam-Rehbruecke (DIfE), 14558 Nuthetal, Germany

5 Research Group Molecular and Clinical Life Science of Metabolic Diseases, Faculty of Health Sciences Brandenburg, University of Potsdam, 14469 Potsdam, Germany

6 Institute of Nutritional Sciences, University of Potsdam, 14558 Nuthetal, Germany

* Correspondence: schuermann@dife.de; Tel.: +49-33-200-88-2368

\begin{abstract}
Pancreatic steatosis associates with $\beta$-cell failure and may participate in the development of type-2-diabetes. Our previous studies have shown that diabetes-susceptible mice accumulate more adipocytes in the pancreas than diabetes-resistant mice. In addition, we have demonstrated that the co-culture of pancreatic islets and adipocytes affect insulin secretion. The aim of this current study was to elucidate if and to what extent pancreas-resident mesenchymal stromal cells (MSCs) with adipogenic progenitor potential differ from the corresponding stromal-type cells of the inguinal white adipose tissue (iWAT). miRNA (miRNome) and mRNA expression (transcriptome) analyses of MSCs isolated by flow cytometry of both tissues revealed 121 differentially expressed miRNAs and 1227 differentially expressed genes (DEGs). Target prediction analysis estimated 510 DEGs to be regulated by 58 differentially expressed miRNAs. Pathway analyses of DEGs and miRNA target genes showed unique transcriptional and miRNA signatures in pancreas (pMSCs) and iWAT MSCs (iwatMSCs), for instance fibrogenic and adipogenic differentiation, respectively. Accordingly, iwatMSCs revealed a higher adipogenic lineage commitment, whereas pMSCs showed an elevated fibrogenesis. As a low degree of adipogenesis was also observed in pMSCs of diabetes-susceptible mice, we conclude that the development of pancreatic steatosis has to be induced by other factors not related to cell-autonomous transcriptomic changes and miRNA-based signals.
\end{abstract}

Keywords: MSCs; fatty pancreas; WAT; lineage commitment; transcriptomics; miRNAs

\section{Introduction}

Obesity, defined as an excess accumulation of white adipose tissue (WAT) mass, is a global health concern that is linked to an increased risk for metabolic perturbations such as type-2-diabetes (T2D), cardiovascular diseases, and non-alcoholic fatty liver disease (NAFLD) [1]. In obesity, WAT expansion occurs through (i) lipid filling within existing fat cells (adipocyte hypertrophy) and (ii) formation of new adipocytes from mesenchymal stromal cells (MSCs) with adipogenic differentiation potential (adipocyte hyperplasia), also well-known as adipogenic precursor cells (APC) [2]. When MSCs exhibit a low ability to differentiate into mature fat-storing adipocytes, the rate of adipocyte hypertrophy is 
increased [2,3]. However, excessive levels of circulating lipids combined with a limited adipocyte storage capacity result in ectopic fat accumulation in non-adipose tissue organs such as liver and muscle, taking the shape of intra-hepatocyte and intra-myocyte lipid droplet stores or as bona fide mature adipocytes interspersed within bone tissue, and the pancreas [4-8]. These ectopic lipid depositions are linked to organ dysfunction, insulin resistance, and the predisposition to develop T2D [4,8]. Even though, the impact of NAFLD on glucose metabolism has been widely studied $[9,10]$, the clinical significance of equivalent fat cells in the pancreas (non-alcoholic fatty pancreas disease, NAFPD) has long been underestimated. Today, there is growing evidence that NAFPD plays a substantial role in human pancreatic fibrosis, pancreatic cancer, and $\beta$-cell dysfunction [11].

Recently, we have reported that genetically diabetes-prone New Zealand Obese (NZO) mice, an obese mouse-strain used as model for polygenic diabetes, exhibit more adipocytes in the pancreas than diabetes-resistant, leptin-deficient B6.V-Lep ob/ob (B6$\mathrm{ob} / \mathrm{ob}$ ) mice [12]. Moreover, co-culture of pancreatic islets and adipocytes derived from MSCs of the pancreas or inguinal white adipose tissue (iWAT) resulted in hypersecretion of insulin [12]. However, the role of pancreas-resident MSCs in modulating organ health is still unclear.

To investigate the function of pancreas MSCs (pMSC) and to compare it with iWAT MSCs (iwatMSCs), we isolated a defined subpopulation of both MSCs from diabetesresistant C57BL/6J (B6) mice using fluorescence-activated cell sorting (FACS) and analyzed their transcriptional and miRNA profiles. MSCs of both sources exhibited marked differences in mRNA and miRNA expression profiles as well as in adipogenic and fibrogenic differentiation capacity.

\section{Results}

\subsection{Distinct Gene Expression Profiles of $p M S C s$ and iwatMSCs}

In order to compare pancreas (pMSCs) and iWAT MSCs (iwatMSCs), we utilized processed pancreases and iWAT of the same male, 8-week-old standard diet fed B6 mice and sorted for a non-endothelial (CD31-), non-hematopoietic (CD45-), adipogenic progenitortype (SCA1+) MSC subpopulation via FACS (Figure 1A,B). Comparative transcriptome analysis of 26,735 expressed transcripts revealed 1,456 differentially expressed transcripts referring to 1227 genes (DEGs) with 757 significantly up- and 699 significantly downregulated genes in pMSCs compared to iwatMSCs (Figure 2A). Gene ontology (GO) enrichment analysis allocated DEGs to 15 significant biological processes (gene count $\geq 10$, fold enrichment $>2$, unadjusted $p<0.05$ ) (Figure $2 \mathrm{~B}$ ), of which most were assigned to positive regulation of developmental processes and fat cell differentiation, cell adhesion, cell migration, and others involving re-arrangement of the cytoskeleton. An enrichment of GO terms including collagen fibril organization, negative regulation of BMP signaling pathway, and negative regulation of fibroblast proliferation pointed toward a potentially alternative fibrogenic cell fate of both types of MSCs. Ingenuity pathway analysis (IPA) identified seven networks, of which one significant pathway linked 11 DEGs directly or indirectly to genes well-known for their role in cell growth and adipogenesis, e.g., Irs-1 (insulin receptor substrates 1) and Irs-2, Pparg (peroxisome proliferator activated receptor gamma), Lepr (leptin receptor) or Srebf (sterol regulatory element-binding transcription factor). This does not only emphasize the strong association of MSCs with the adipogenic lineage but the versatile developmental nature of mesenchymal-type precursor cells (Figure 2C). 
A

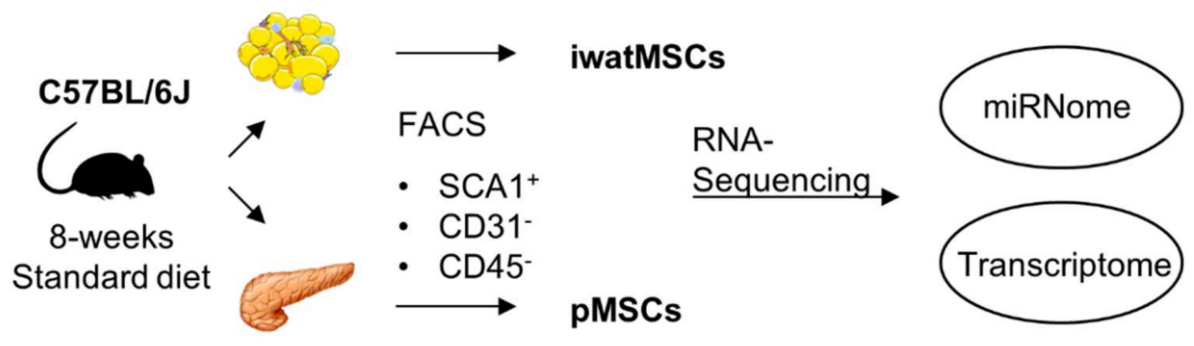

B
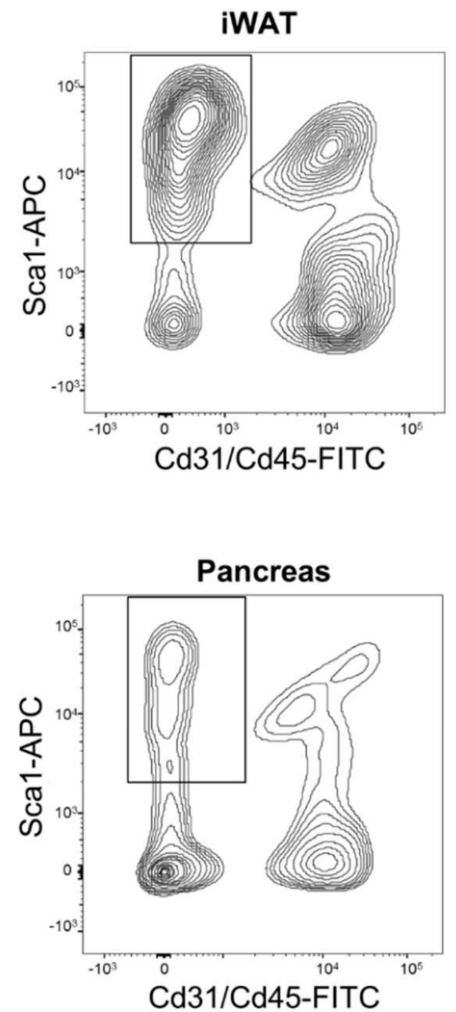

C
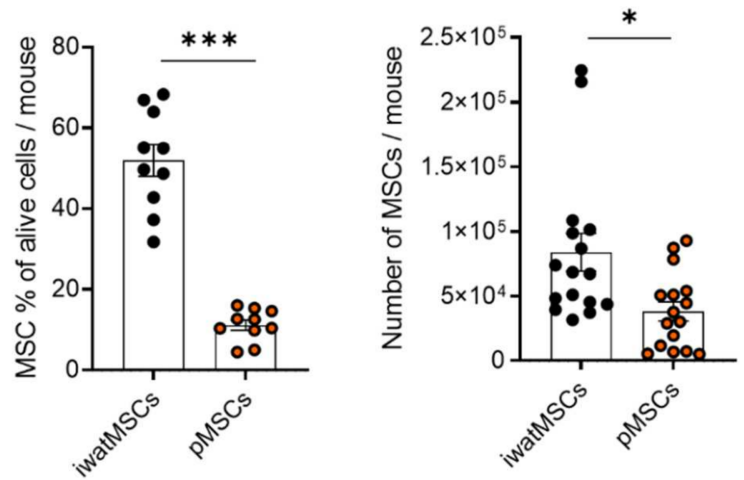

D

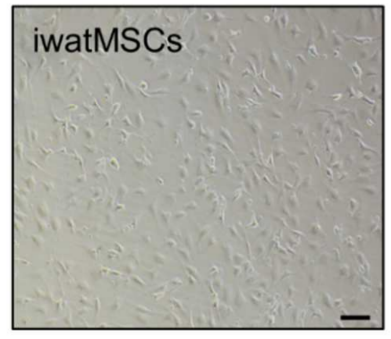

Figure 1. Isolation of mesenchymal stromal cells from inguinal white adipose tissue and pancreas for transcriptome and miRNome analyses. (A) Study design. iWAT and pancreas were collected from the same C57BL/6J mice and MSCs were isolated via fluorescence-activated cell sorting (FACS). After expansion, iwatMSCs and pMSCs were harvested and transcriptome and miRNome analyses were performed. (B) FACS of cells isolated from iWAT and pancreas. Box marks the non-endothelial (CD31-), non-hematopoietic (CD45-), adipogenic progenitor (SCA1+) MSC subpopulation. (C) Graphical representation of alive iwatMSC (black) and pMSC (orange) fraction per mouse and the total sorted iwatMSC and pMSC number per mouse. (D) Representative microscopic images of iwatMSCs and pMSCs after 4 days of expansion on cell culture plates. Scale bars: $100 \mu \mathrm{m}$. Differential expression defined by Student's $t$-test with Welch's correction unadjusted ${ }^{*} p<0.05,{ }^{* * *} p<0.0001$. 
A

Down in pMSCs Up in pMSCs

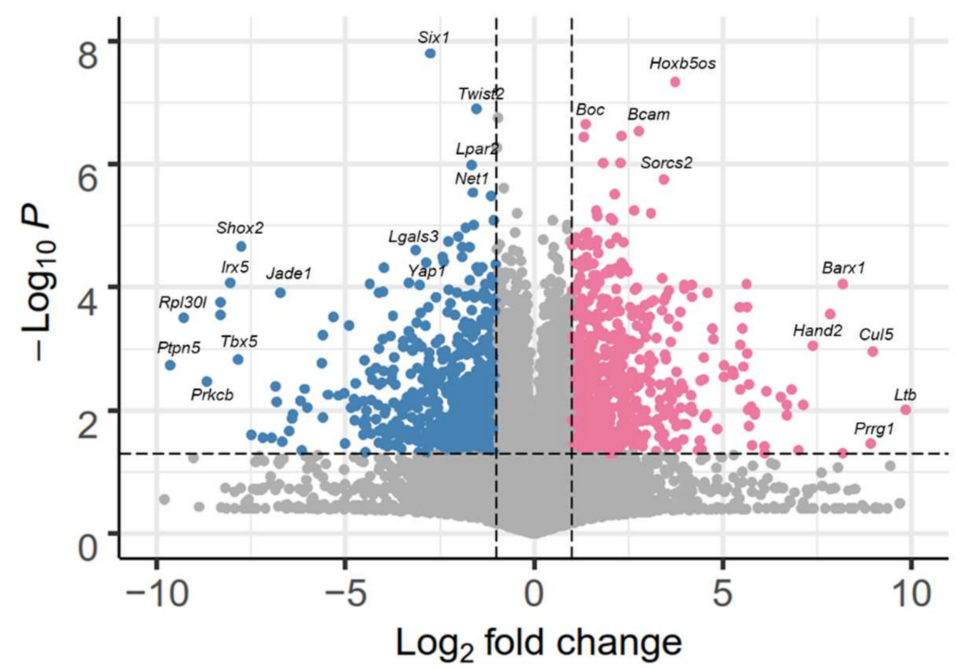

B

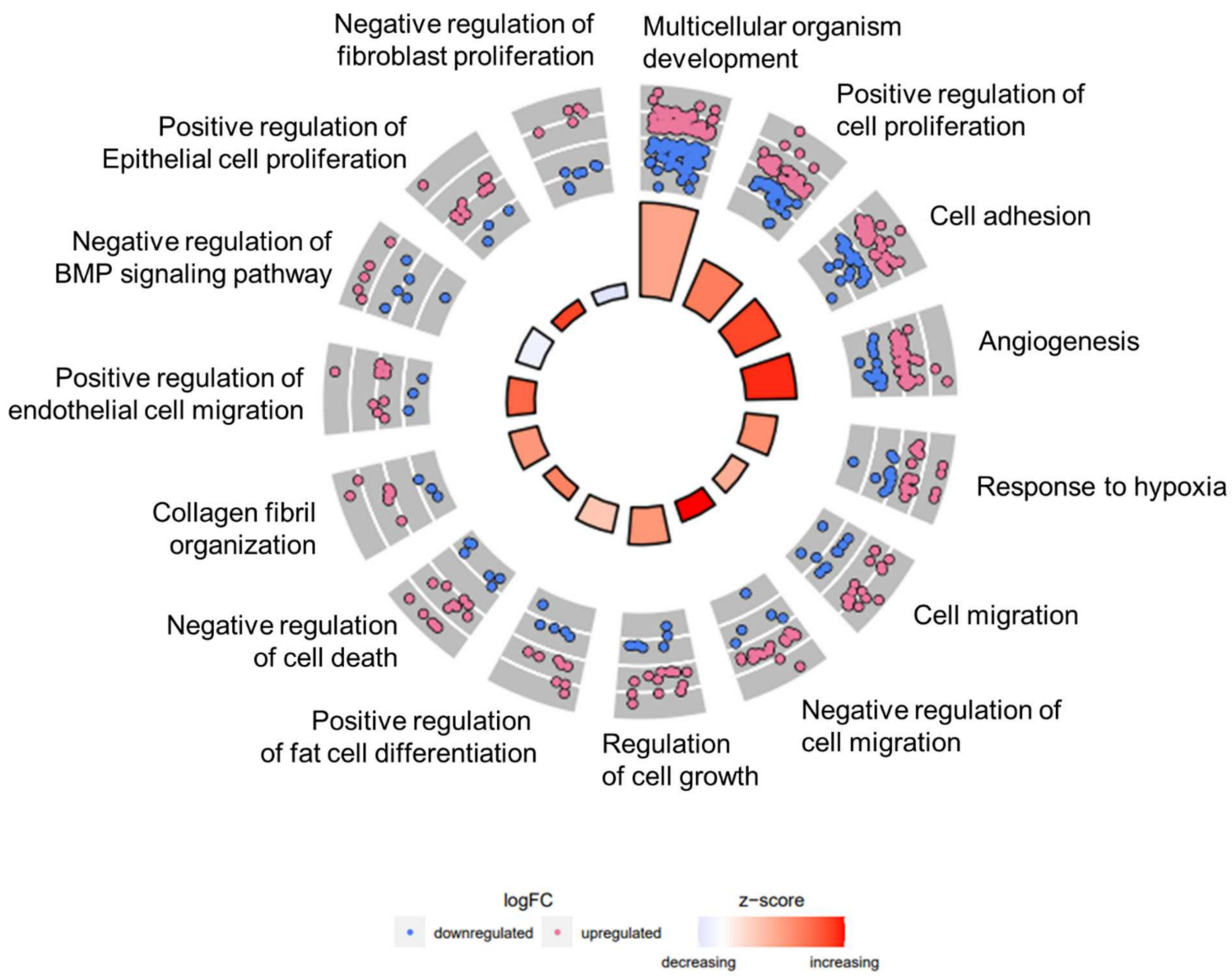

Figure 2. Cont. 
C

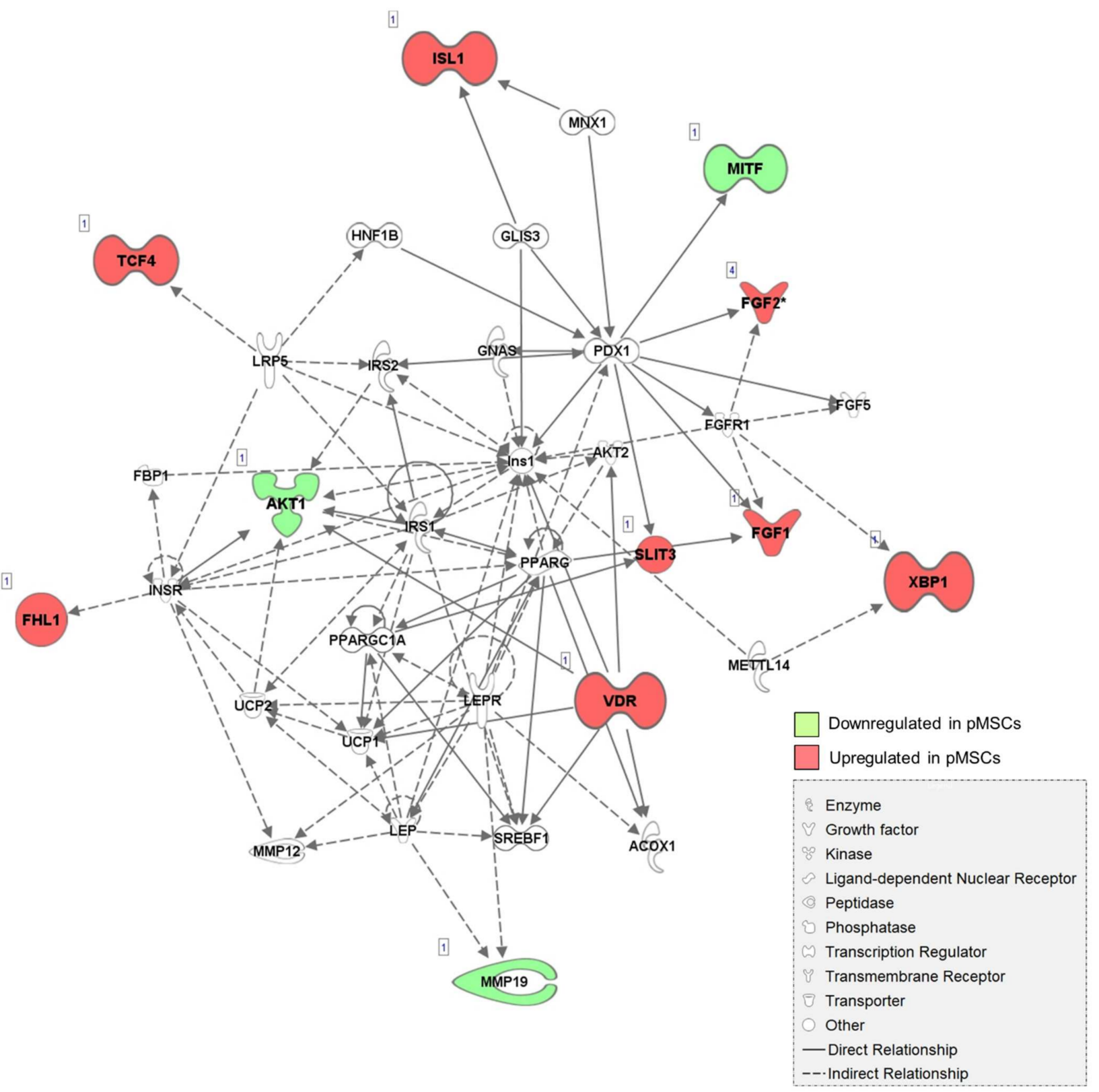

Figure 2. Comparative transcriptome and pathway analyses show various distinct biological processes between pMSCs and iwatMSCs. (A) Volcano plot of 26,735 expressed transcripts in iwatMSCs and pMSCs. Blue and pink dots depict up- and down-regulated transcripts in pMSCs, respectively $(n=8$; Student's $t$-test with Welch's correction unadjusted $p<0.05)$. (B) Circular visualization of gene-ontology enrichment analysis (GOcircle) with 1227 genes that differ in their expression between iwatMSCs and pMSCs. The inner circle indicates the main biological processes to be either increased (red) or decreased (blue) in pMSCs (shown by the z-score). The outer circle depicts scatter plots for genes allocated to the most significant biological processes and the direction of regulation in pMSCs. Blue dots mark down-regulated and red dots up-regulated genes in pMSCs. (C) Gene expression network identified by Ingenuity Pathway Analysis (IPA). The network links 11 of the DEGs to genes known for cell-growth and adipogenesis. Down-regulated genes in pMSCs are shown in green and up-regulated genes are marked in red. 
Proteins that are actively transported within the secretory pathway play a pivotal role in the regulation of critical cellular functions and are defined as "secreted proteins". A majority of these proteins, including cytokines, growth factors, and hormones, participate in local and systemic signaling functions [13]. Therefore, an in-silico secretome analysis was performed using the Human Protein Atlas. Interestingly, 154 of the 1227 DEGs were estimated to encode for secreted proteins, of which 112 genes were higher expressed in pMSCs and 42 in iwatMSCs (Figure 3, Supplementary Table S2B). To gain insight into functional processes of potentially secreted proteins, we looked at the top 10 predicted secreted proteins either abundant in pMSCs or iwatMSCs, respectively. Genes of potentially secreted proteins in iwatMSCs were mainly allocated to cell proliferation (Prl, Grem2, Sfrp2) and angiogenic processes (Mmp2, Mmp19, Thbs2). In contrast, transcripts of secretory proteins in pMSCs were assigned to proliferative events (Igfbp4, Igfbp7, Smoc2, Slit3) and immunomodulation (Cxcl2, Angptl2, Gpx3) (Figure 3).

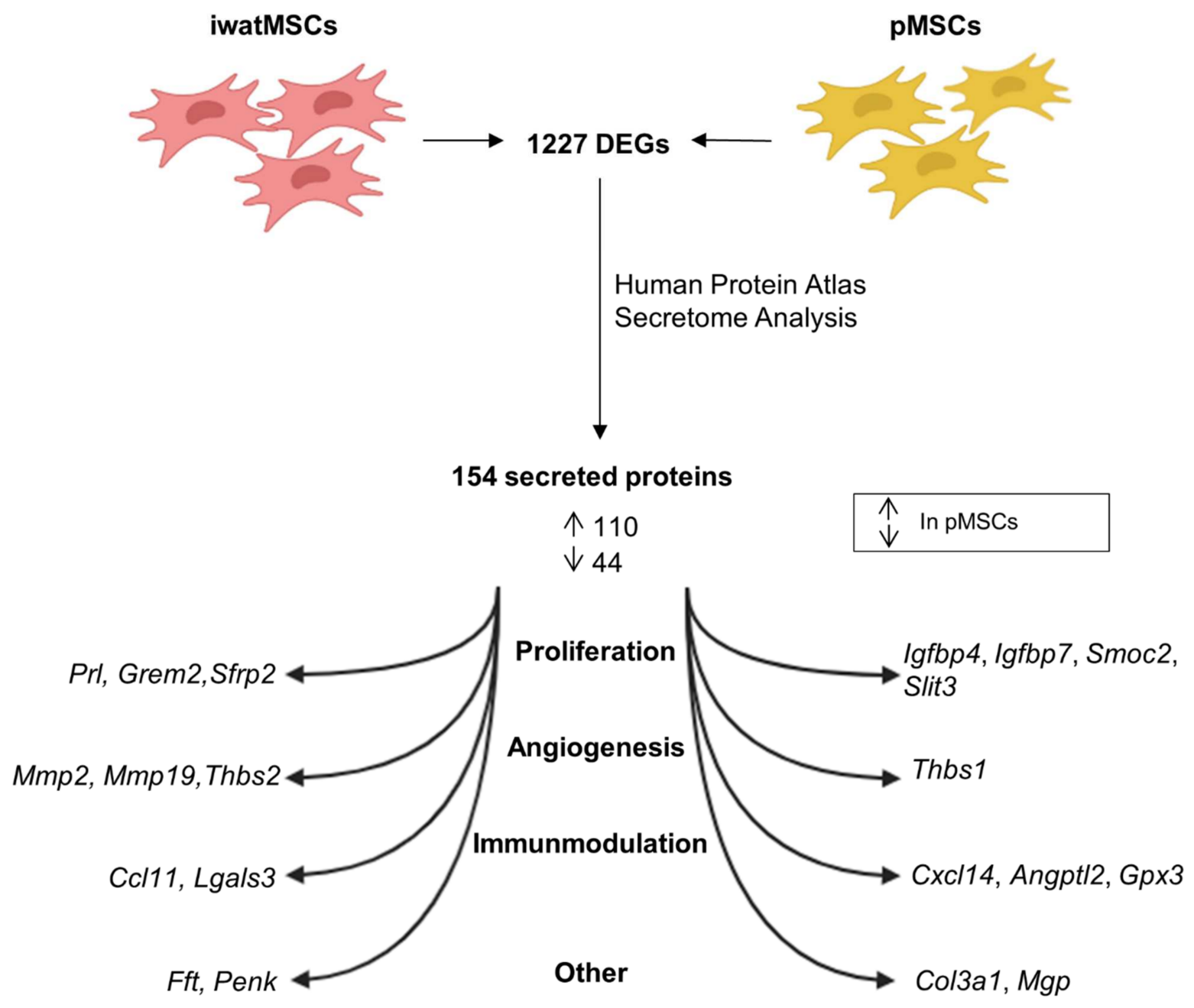

Figure 3. In-silico secretome analysis predicts a higher number of secreted proteins in pMSCs compared to iwatMSCs. The 1227 DEGs between iwatMSCs and pMSCs MSCs were used to identify putatively secreted proteins via Human Protein Atlas. The in-silico secretome analysis is based on expression patterns and predicted 154 secreted proteins among the DEGs, of which 110 might be secreted by pMSCs and 44 by iwatMSCs. Shown are top-ten protein-coding genes predicted to be mainly secreted by iwatMSCs and pMSCs and their putative function, respectively.

\section{2. miRNA Expression Patterns of $p M S C s$ and iwatMSCs}

miRNAs are short, 19-23 nucleotides long single-stranded RNA sequences playing a critical role in the post-transcriptional regulation of gene expression. They act in functional 
networks as each miRNA targets a few to several mRNAs [14]. Expression analysis of 1240 miRNAs in pMSCs and iwatMSCs revealed that 121 were differentially expressed, of which 66 were higher and 55 lower expressed in pMSCs compared to iwatMSCs (Figure 4A). To link differences in miRNA expression to altered mRNA levels, we performed a target gene prediction analysis and identified 58 differentially expressed miRNAs putatively regulating 510 genes (Supplementary Table S2C,D). As miRNAs usually negatively regulate gene expression, only target genes were considered that fit to the direction of miRNA expression (e.g., miRNA expression up, target gene expression down). Next, GO enrichment analyses were performed with predicted target genes for pMSCs and iwatMSCs, separately (gene count $\geq 3$, fold enrichment $>2$, unadjusted $p<0.05$ ) (Figure 4B,C). In fact, comparison of both GO-circles showed no overlaps between the depicted biological processes, indicating key differences in miRNA-regulated gene expression in both MSC types. While miRNA targets of pMSCs are predominantly involved in transcription and gene expression processes, iwatMSCs are mainly linked to cytoskeleton-related pathways and proliferation.

Furthermore, both MSC populations differ in their miRNA expression signatures: While miR-204-5p, miR-211-5p, miR-196a-5p, and miR-196b-5p were almost exclusively expressed in iwatMSCs (Figure 4D), miR-335-5p, miR-375-3p, miR-429-3p, and miR-200a-3p were mainly detected in pMSCs (Figure 4E). For miR-204-5p we determined 41 regulated genes (Supplementary Table S2C,D) with a higher expression in pMSCs than in iwatMSCs. The strongest repression with log2-fold changes of $>1.5$ were observed for those candidates shown in the box of Figure 4D. Among these suppressed genes is the ephrin receptor gene, Ephb2, which can also act as a ligand and regulates diverse cellular processes including motility, division, and differentiation [15]. Other targets are Dnajc14, a heat shock protein; Slc7a2, a cationic amino acid transporter; Arhgef5, a Rho guanine nucleotide exchange factor, pointing toward a function in controlling cytoskeletal organization. Theoretically, miR-211$5 p$ targets three genes (Tcf4, Cux1, Trps1) which exhibit a lower expression in iwatMSCs than in pMSCs. However, only Trps1, a transcriptional repressor, reaches the threshold of $>1.5$-fold differential expression (see box in Figure 4D). miR-196a-5p and miR-196b-5p both target the phospholipase A2 receptor 1 (Pla2r1), even though the log2-fold changes here were $<1.5$ (Supplementary Table S2D). This gene is described to promote cellular senescence by inducing DNA damage [16]. Thus, via an elevated miR-196a/b expression iwatMSCs might lower Pla2r1 levels and protect against senescence.

The pMSC-abundant miRNA miR-335-5p, theoretically targets 32 genes which show a lower expression in comparison to iwatMSCs. Among these, 13 genes exhibit a repression with $\log 2$-fold changes of $>1.5$ (see box in Figure 4E). Met (mesenchymal endosomal transition) encodes a receptor tyrosine kinase, which is activated by hepatocyte growth factor (HGF) and thereby involved in the regulation of a variety of biological processes including cell motility, cell proliferation, the epithelial-to-mesenchymal transition, and the development and progression of cancer cells [17]. Another interesting target is Hmgcr (3hydroxy-3-methylglutaryl-coenzyme A reductase), the rate-limiting enzyme for cholesterol synthesis. miR-375-3p targets Yap1 (yes-associated protein 1) with a threshold of $>1.5$. This gene encodes an oncogene, a downstream nuclear effector of the Hippo signaling pathway. In its unphosphorylated state, Yap1 translocates to the nucleus where it binds to TEAD family transcriptions factors and regulates genes promoting proliferation [18]. miR-429-3p is thought to target the glycosyltransferase St3gal2. Deletion of this gene induces late onset of obesity and insulin resistance, particularly in adipose tissue, which was associated with altered ganglioside profiles [19]. miR-200a-3p targets Pafah1b1 (platelet activating factor acetylhydrolase $1 \mathrm{~b}$ regulatory subunit 1 ), which causes type I lissencephaly, a neuronal migration disorder, upon haploinsufficiency [20]. In mice, genetic deletion of Pafah1b1 in the liver attenuated lipid release and thereby increased fat accumulation in the hepatocytes [14]. 


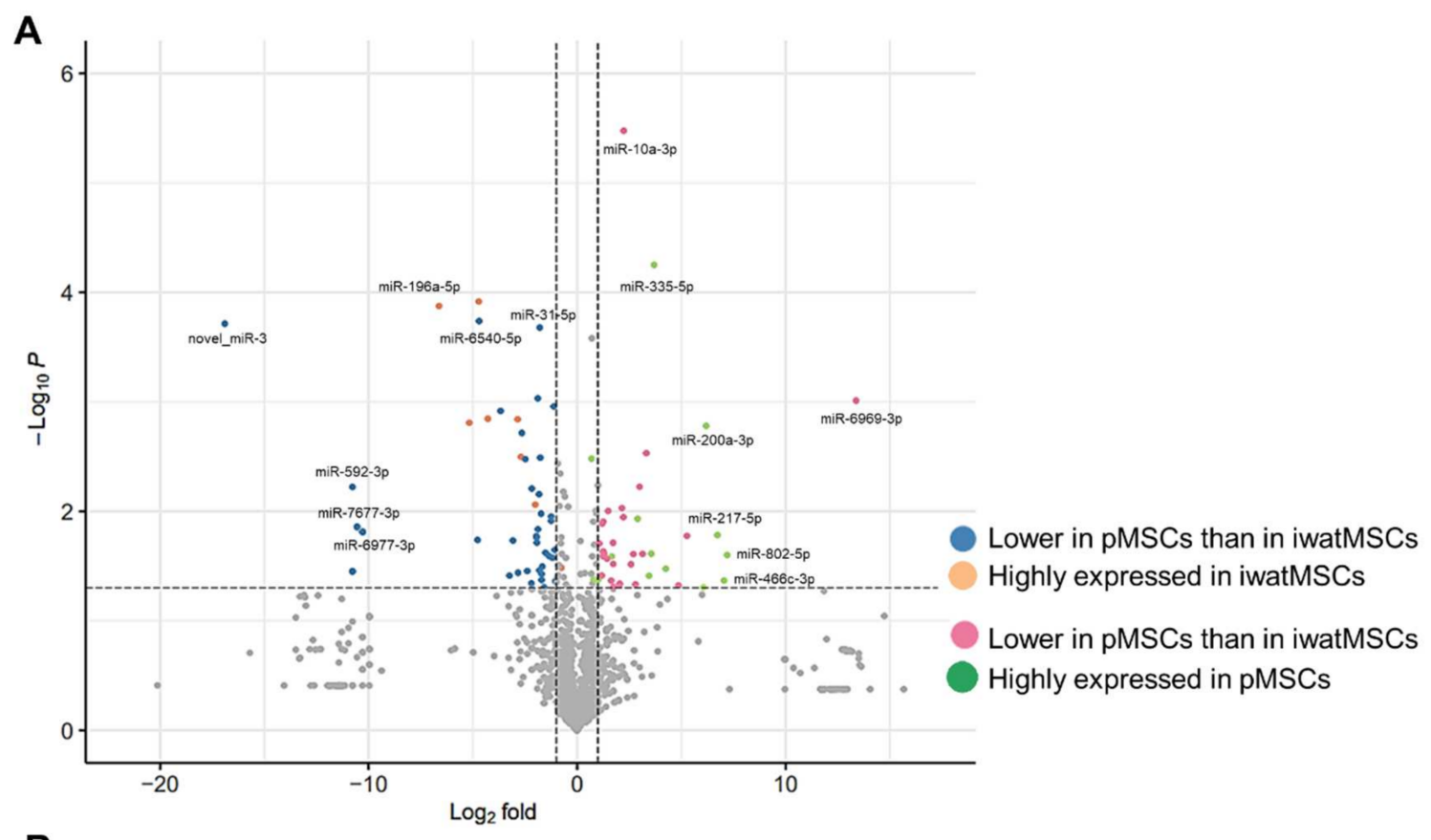

B

\section{iwatMSC miRNA target genes}

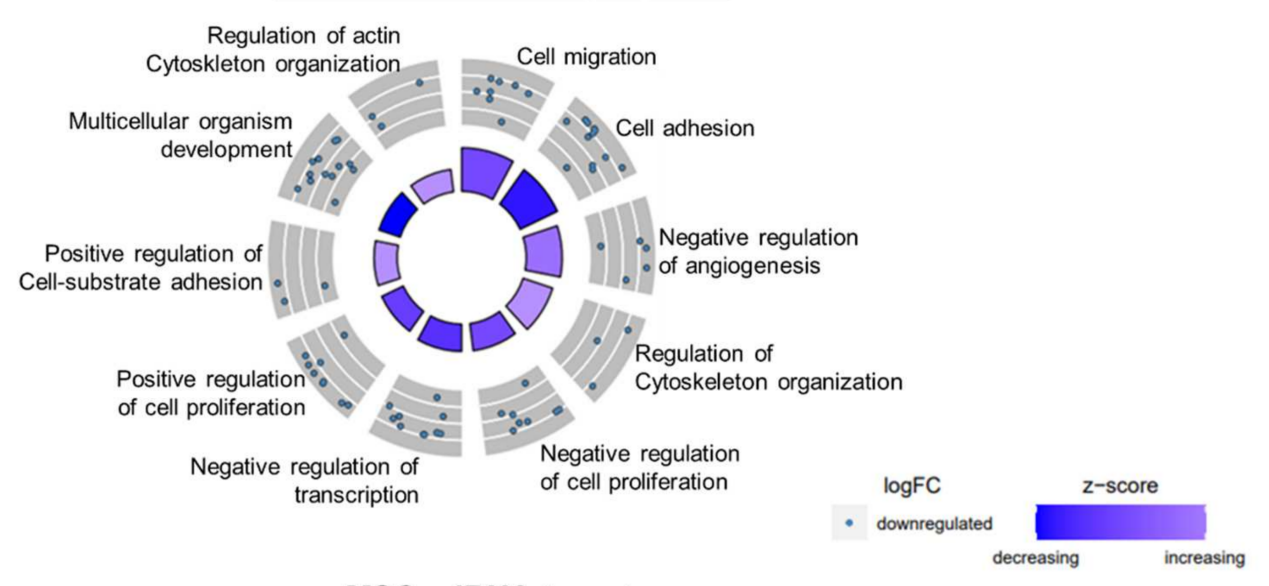

C

\section{pMSC miRNA target genes}

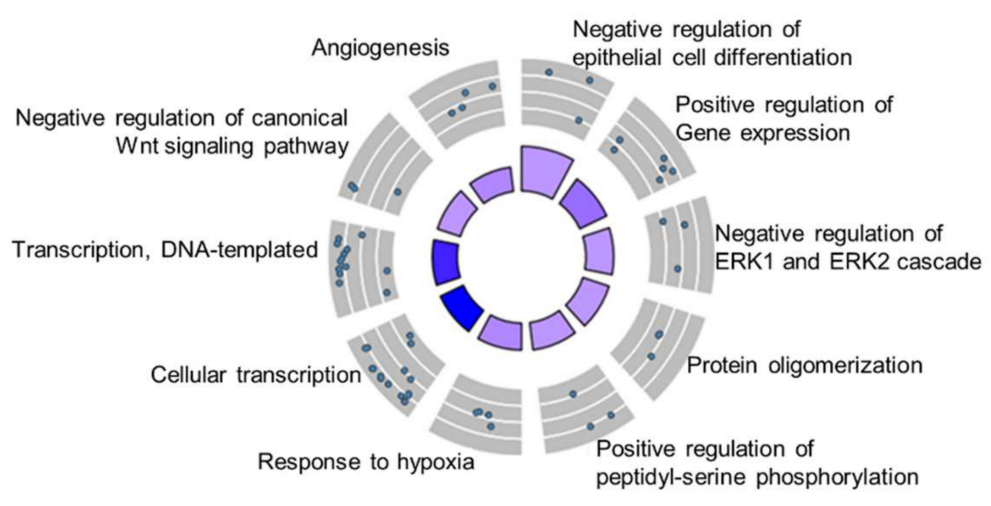

Figure 4. Cont. 


\section{D}

miR-204-5p

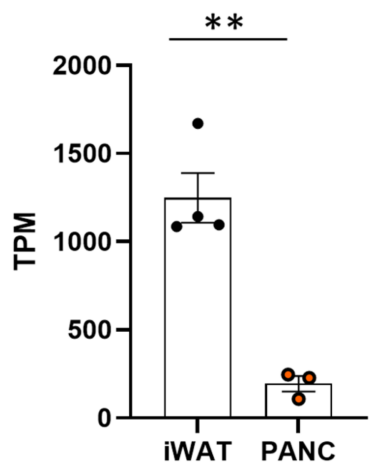

Ephb2, Sgcd, Pdlim2,

Dnajc14, Jade1, Slc7a2,

Arhgef5, Trps1, Nexn,

Prss23, Ubr4, Myl9, Zbtb34

E

miR-335-5p

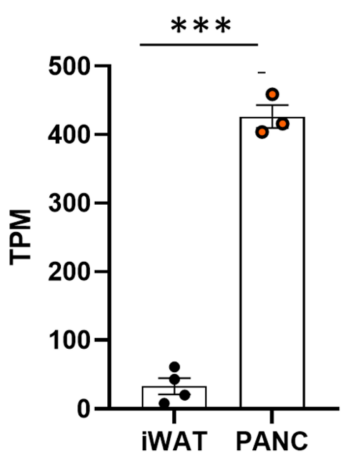

Cav1, Met, Dcakd, Hmgcr, Naa50, Jade1, Shox2, Sh3bgrl2, TIr4, Nhsl1, Cpne8, Ubr4, Atf7
miR-211-5p

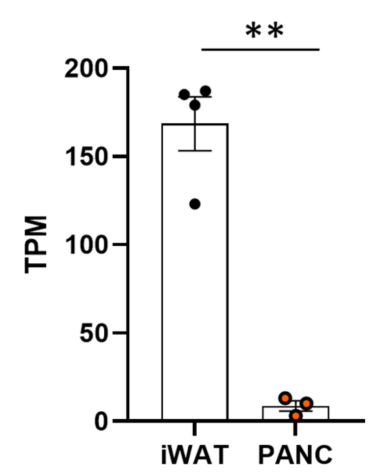

Trps1
miR-196a-5p

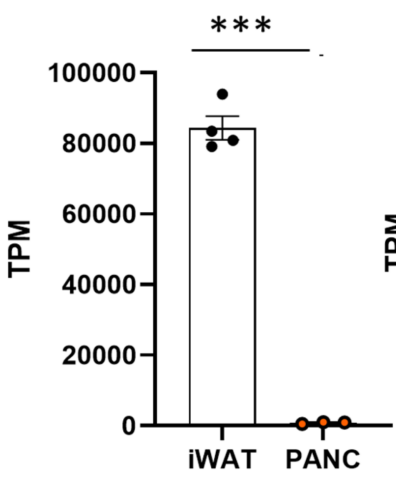

miR-196b-5p

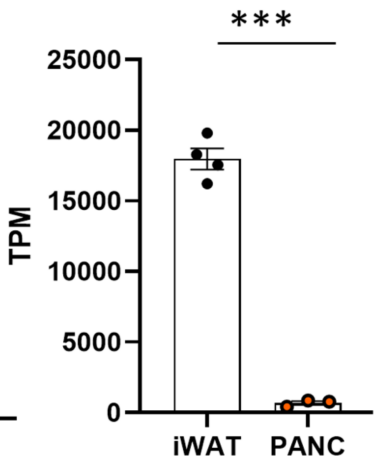

miR-375-3p

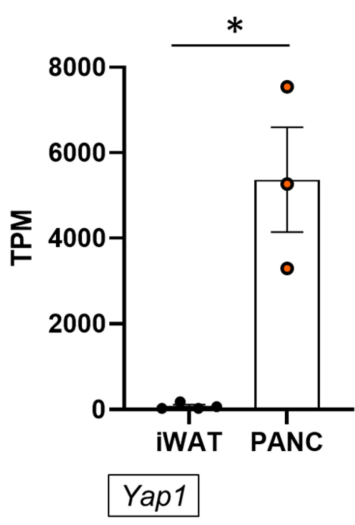

miR-429-3p

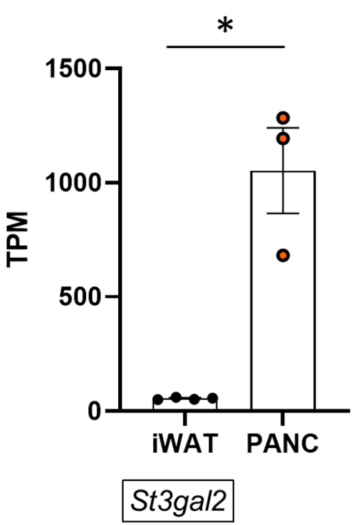

miR-200a-3p

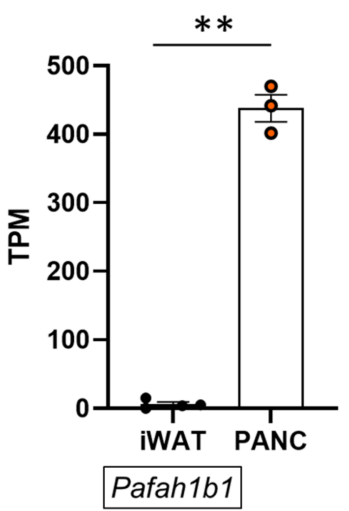

Figure 4. Differentially expressed miRNA between iwatMSCs (iWAT) and pMSCs (PANC) might participate in their distinct expression profiles. (A) Volcano plot of 1240 expressed miRNAs showing differences between iwatMSCs versus pMSCs. 66 miRNAs were higher expressed (blue and orange dots), while 55 were lower expressed (pink and green dots) in pMSCs than in iwatMSCs. Green dots highlight miRNA which are predominantly expressed in iwatMSCs, whereas orange dots show miRNA mainly regulated in pMSCs (defined by $p \leq 0.05$ and $\log 2 \mathrm{FC} \geq 2)$. $(n=8$; Student's $t$-test with Welch's correction unadjusted $p<0.05)$. (B,C) Gene ontology (GO) enrichment analyses with predicted target genes of highly expressed miRNAs in iwatMSCs and pMSCs (defined by $p \leq 0.05$ and $\log 2 \mathrm{FC} \geq 2$ ), respectively. The GOcircle plots show the top 10 enriched GO-terms in the category biological processes. The inner circle reflects the direction of the indicated biological processes (dark blue $=$ increased, light blue $=$ decreased). The outer circle depicts scatter plots for miRNA target genes allocated to the respective biological processes. (D,E) miRNA nearly exclusively expressed in iwatMSCs and pMSCs, respectively. Target genes with a repression of log2FC $>1.5$ are shown accordingly in boxes $\left({ }^{*} p<0.05,{ }^{* *} p<0.005,{ }^{* * *} p<0.0001\right)$. 


\subsection{Decreased Adipogenic Potential of $p M S C s$}

As a large number of mRNAs and miRNAs differed in their abundance in pMSCs and iwatMSCs and depot-specific differences with regard to the adipogenic capacity are well-known $[7,21]$, we tested if they varied in their lineage commitments by performing differentiation assays. To this end, pMSCs and iwatMSCs from B6 mice were subjected to a specific adipocyte differentiation protocol or incubated without the differentiation cocktail to evaluate the levels of spontaneous adipogenesis. The iwatMSCs showed some degree of spontaneous differentiation to adipocytes and adipogenesis was observed in almost all cells following the adipogenic induction treatment. In contrast, pMSCs exhibited a markedly decreased spontaneous as well as induced adipogenesis compared to iwatMSCs as reflected by a lower number of Oil Red O-positive cells (Figure 5A). Quantification of the lipid droplets showed an almost $70 \%$ lower adipogenic potential after adipogenesis induction in pMSCs (Figure 5B). In line with this, expression levels of adipocyte marker genes like the transcription factors Cebpa (CCAAT/enhancer-binding protein alpha) and Pparg (peroxisome proliferator-activated receptor gamma) as well as Adipoq (the adipokine adiponectin) and Plin1 (lipid-droplet associated protein perilipin 1) were 4-to 9-fold lower expressed in pMSCs in comparison to iwatMSCs (Figure 5C). Remarkably, mRNA levels of Slc2a4 (glucose transporter 4) were close to the detection limit in pMSCs.

\subsection{Increased Fibrogenic Potential in $p M S C s$}

As the adipogenic potential of pMSCs was significantly lower than that of iwatMSCs, we hypothesized an alternative cell fate programming for pMSCs. Our expression analysis of transcripts of putatively secreted proteins (Figure 2B) pointed toward a potential fibrogenic lineage commitment. Therefore, we tested the fibrogenic capacity by treating pMSCs and iwatMSCs with TGF- $\beta 1$ followed by staining of fibrogenesis marker $\alpha$-SMA. The representative pictures (Figure 6A) and the morphometric analysis displayed significantly higher $\alpha$-SMA staining intensity in pMSCs (Figure 6B) supporting a higher capacity to undergo fibrogenesis compared to iwatMSCs. In agreement with this, expression levels of representative fibrogenesis marker genes like Acta2 (actin alpha 2), Col1a1 (collagen type1 alpha 1) and Pdgfra (platelet-derived growth factor receptor A) were higher expressed in pMSCs than in iwatMSCs (Figure 6C).

To test whether the implied fibrogenic development of pMSCs affected insulin secretion, glucose-stimulated insulin secretion experiments were performed with isolated pancreatic islets of $\mathrm{B} 6$ mice alone or with islets that were co-cultured with fibrogenically differentiated, i.e., TGF- $\beta 1$-treated, pMSCs. In fact, insulin secretion did not differ between both conditions. It was increased in response to $20 \mathrm{mM}$ glucose by 6-7-fold in the presence or absence of fibrotic cells. Thus, acute fibrogenesis of MSCs in the pancreas does not impair insulin secretion (Figure 6D).

\subsection{Similar Cell-Fate Decisions in pMSCs Derived from Diabetes-Susceptible and Diabetes-Resistant Mice}

As we have previously shown that diabetes-prone NZO mice exhibit increased amounts of fat cells in their pancreas, while diabetes-resistant B6-ob / ob have nearly no fat cells in this organ, we reasoned that $\mathrm{pMSC}$ derived from NZO mice might show a more pronounced adipogenic cell fate commitment. Therefore, pMSCs and iwatMSCs were isolated from 8-week-old B6 and NZO mice (as described in Figure 1). When comparing the sorted cell counts normalized to tissue mass, we did not identify any significant differences between the two mouse strains (Supplementary Figure S1). Next, an expansion period of 4 days was followed by the induction of adipogenesis or fibrogenesis. As before, adipogenic potential of pMSCs was limited in comparison to that of iwatMSCs derived from both B6 and NZO mice (Figure 7A,B). In line with this, pMSCs of B6 and NZO displayed an increased $\alpha$-SMA signal intensity compared to iwatMSCs. Again, no strain-specific differences were found (Figure 7C,D). These findings clearly show that factors other than cell-autonomous ele- 
ments of pMSCs, e.g., endocrine signals from liver or other tissues, may induce adipocyte accumulation in pancreas of diabetes-susceptible NZO mice.

A

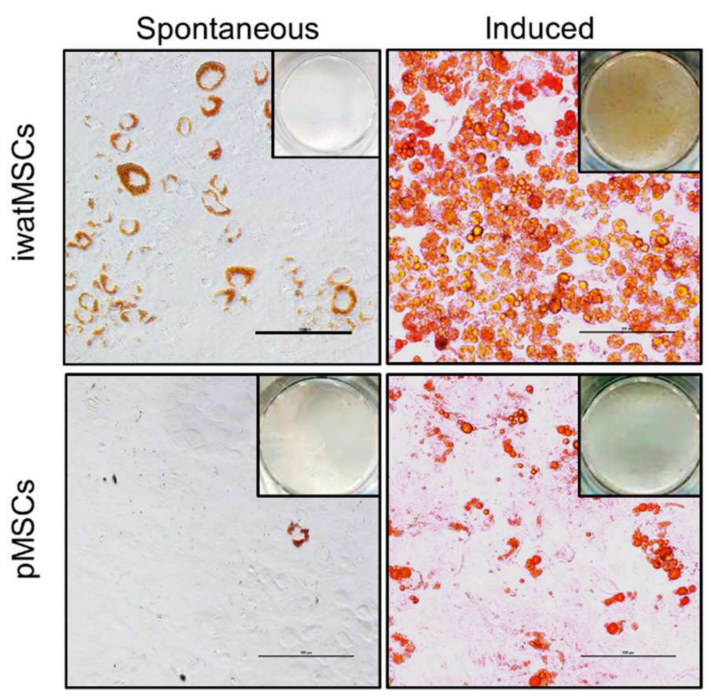

B

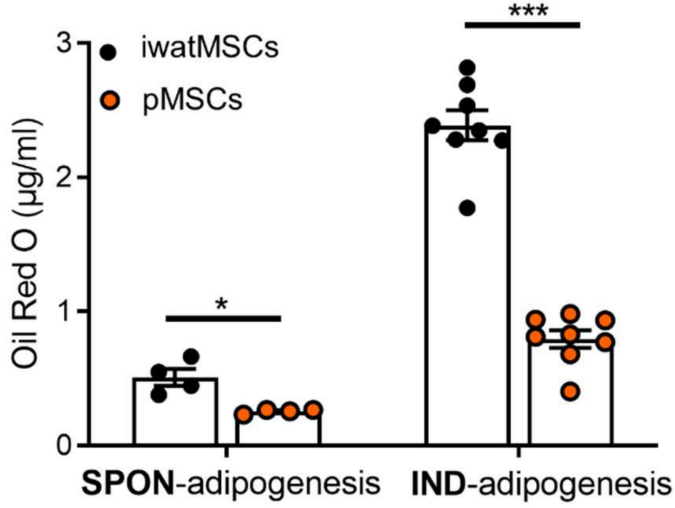

C

- iwatMSCs o pMSCs

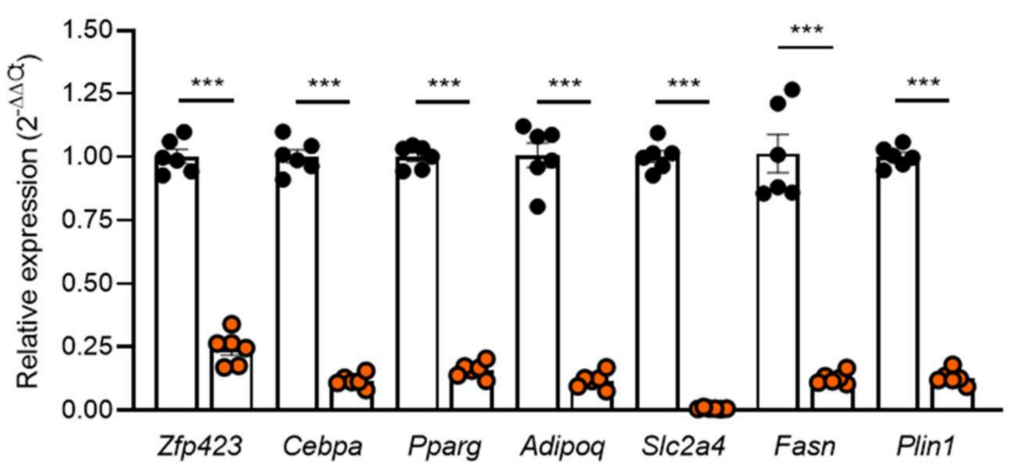

Figure 5. pMSCs display a significantly reduced adipogenesis in comparison to iwatMSCs. After flow-cytometric sorting, iwatMSCs and pMSCs were seeded on cell culture plates and expanded for 4 days. After an adipogenic induction of 2 days, cells were differentiated for another 10 days before analysis. (A) Representative images of Oil Red O stainings in iwatMSCs and pMSCs showing spontaneous (SPON-) and induced (IND-) adipogenesis. Scale bars: $100 \mu \mathrm{m}$. (B) Quantification of Oil Red O staining ( $n=4-8$ per group). (C) Relative expression of indicated adipogenesis markers in iwatMSCs and pMSCs after treatment with the differentiation cocktail ( $n=5-6$ per group). Data shown as mean \pm SEM. Statistical difference was calculated using Student's $t$-test with Welch's correction $\left({ }^{*} p<0.05,{ }^{* * *} p<0.001\right)$. 


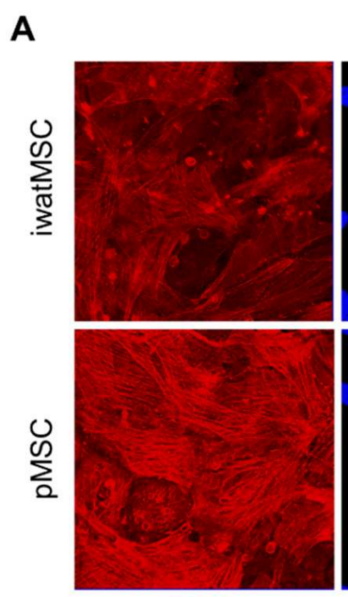

aSMA
Fibrogenesis

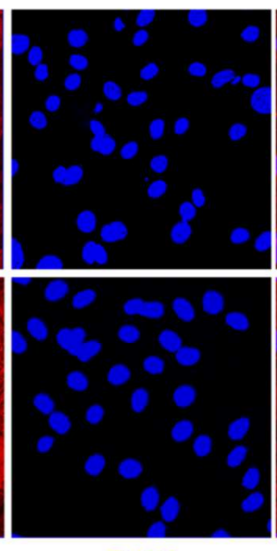

DAPI
B

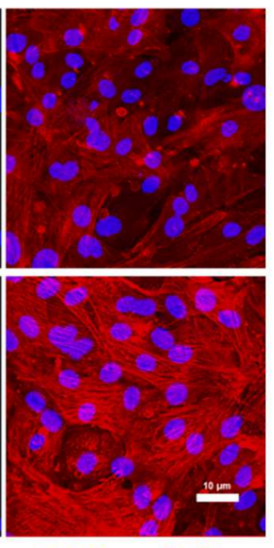

DAPI + aSMA

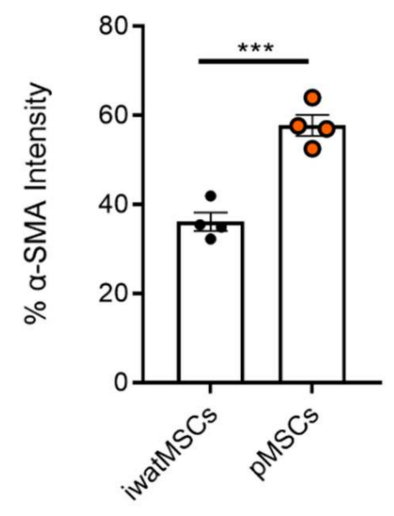

C

- iwatMSCs

- pMSCs

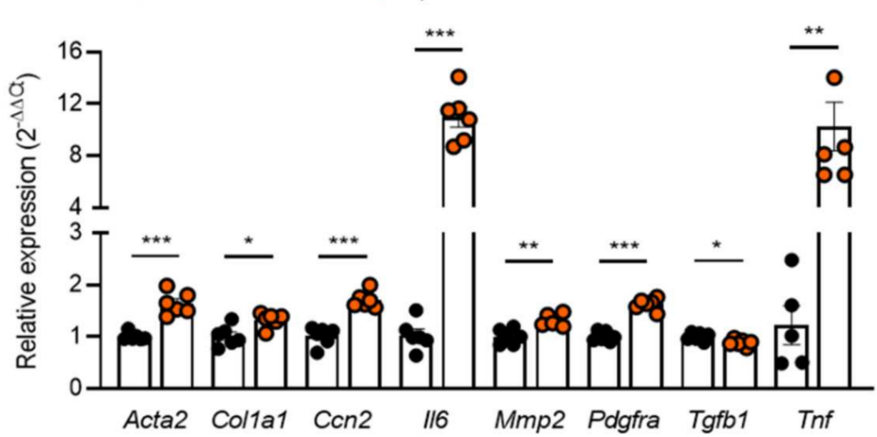

D

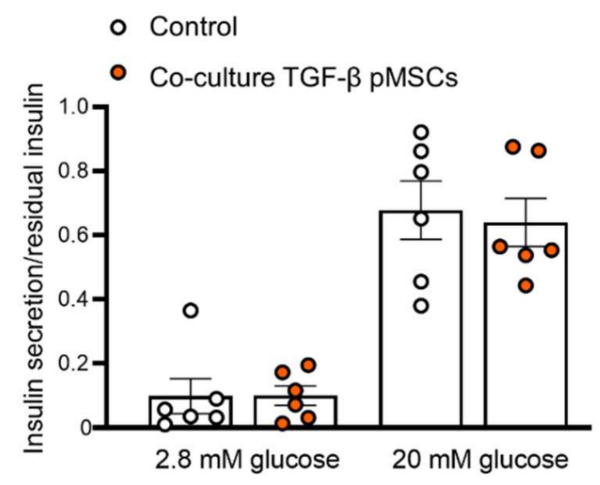

Figure 6. pMSCs exhibit increased levels of the fibrosis-marker $\alpha$-SMA. Sorted and expanded (4 days) pMSCs and iwatMSCs were treated with TGF- $\beta 1$ for 5 days to induce fibrogenesis. (A) Representative images of iwatMSCs and pMSCs stained for the fibrogenic marker $\alpha$-SMA (red). Nuclei were detected with DAPI (blue). DAPI: 1200 per group, scale bars: $40 \mu \mathrm{m}$. (B) Morphometric analysis of $\alpha$-SMA signal intensity ( $n=4-8$ per group). (C) Relative expression levels of indicated fibrogenesis markers in iwatMSCs and pMSCs after TGF- $\beta 1$ treatment ( $n=6$ per group). (D) Glucose-stimulated insulin secretion of pancreatic islets of B6 mice alone or co-cultured with TGF- $\beta 1$ treated pMSCs. ${ }^{*} p<0.05$, ** $p<0.005,{ }^{* * *} p<0.001$. 
A

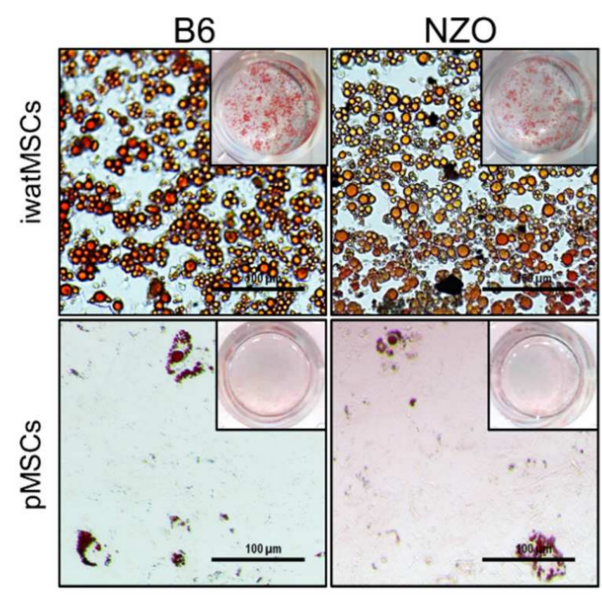

C

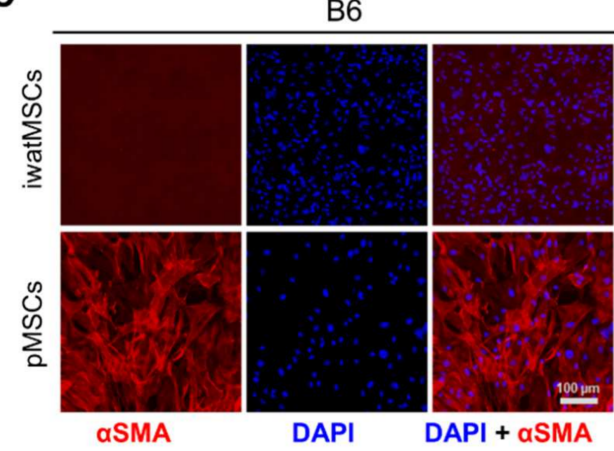

B

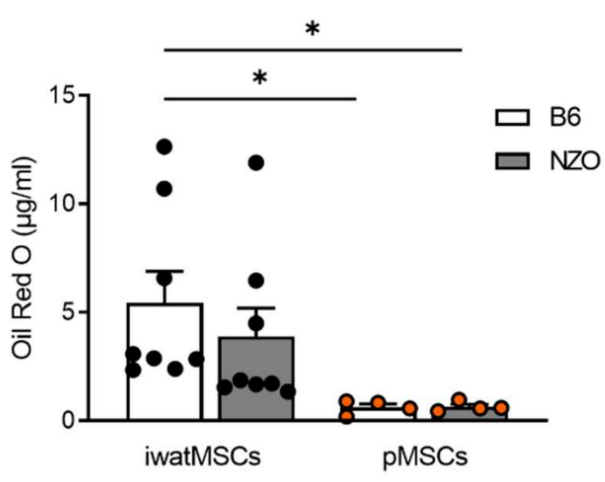

$\mathrm{NZO}$

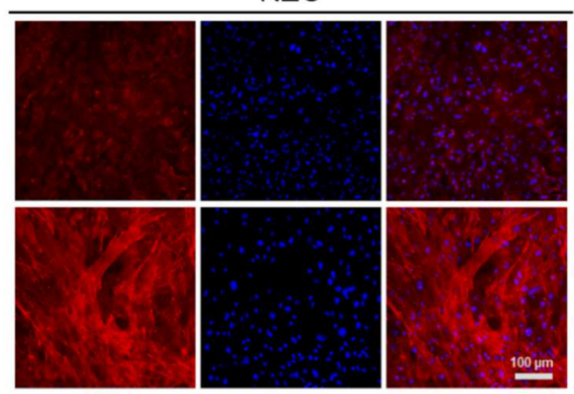

DAPI

DAPI + aSMA

D

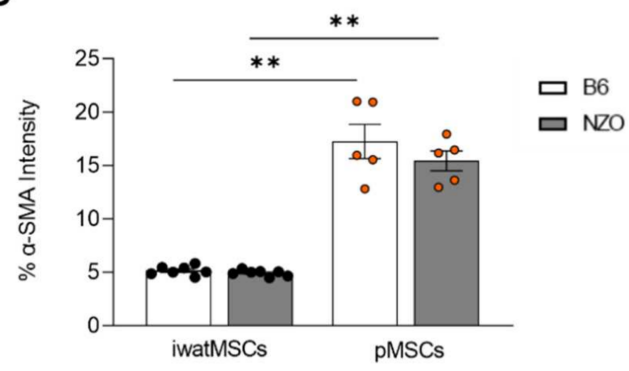

Figure 7. pMSCs and iwatMSCs of diabetes-susceptible NZO mice display similar lineage commitments as shown for B6 animals. (A) After flow-cytometric sorting, iwatMSCs and pMSCs of B6 and NZO mice were seeded on cell culture plates and expanded for 4 days. After an adipogenic induction of 2 days, cells were differentiated for another 10 days before analysis. Representative images of Oil Red O stainings in iwatMSCs and pMSCs of both mouse strains showing induced adipogenesis. Scale bars: $100 \mu \mathrm{m}$. (B) Quantification of Oil Red O stainings ( $n=5$ per group). (C) For analysis of the fibrogenic differentiation capacity, iwatMSCs and pMSCs were treated with TGF- $\beta 1$ for 5 days. Representative images of iwatMSCs and pMSCs of B6 and NZO mice stained for the fibrogenic marker $\alpha$-SMA (red). Nuclei were detected with DAPI (blue). DAPI: 1200 per group, scale bars: $40 \mu \mathrm{m}$. (D) Morphometric analysis of $\alpha$-SMA signal intensity ( $n=5$ per group). Data shown as mean \pm SEM. Statistical difference was calculated using Student's $t$-test with Welch's correction (* $p<0.01$, $\left.{ }^{* *} p<0.05\right)$. 


\section{Discussion}

Mesenchymal stromal cells (MSCs) are multipotent stem cells present in the stroma of many tissues and can give rise to a number of cells including adipocytes [22]. Our study identified marked variations in the expression profile and differentiation characteristics between MSCs isolated from pancreas (pMSCs) and iWAT (iwatMSCs). In general, MSCs are able to differentiate into several lineages, e.g., adipocytes and fibroblasts [3,23]. As expected, iwatMSCs exhibit a major potential to differentiate into adipocytes. In contrast, despite sorting cells with the same set of surface marker proteins, pMSCs preferentially differentiated into fibrogenic rather than adipogenic cells. Considering that spontaneous adipogenic differentiation can impede the differentiation potential of preadipocytes into other lineages [24], the lower spontaneous adipogenic capacity of pMSCs compared to iwatMSCs which we observed in our functional analyses agrees with the increased fibrogenic cell-fate of pMSCs.

Interestingly, also pMSCs of diabetes-susceptible NZO mice, in which we previously detected a high number of adipocytes in pancreas, exhibit a low degree to differentiate into adipocytes [12]. However, beside NZO mice, another T2D mouse model has been described to develop fat infiltrations in the pancreas [25]. Thus, we hypothesize that other species-specific factors are responsible for the adipocyte accumulation in pancreas.

The distinctive cell lineage fates of both MSC populations are clearly reflected by the transcriptome. In pMSCs, enriched genes are linked to a function in cytoskeleton, cell growth, and particularly in negative regulation of BMP signaling and fibroblast proliferation, indicating their elevated fibrogenic cell fate. In fact, our in vitro studies showed the differentiation of pMSCs to fibrogenic cells which might indicate that pMSCs play a role in fibrosis of the pancreas, e.g., in the development of pancreatitis. Due to the expression of genes related to a positive regulation of fat cell differentiation, pMSCs are principally able to become adipocytes. The IPA (Ingenuity Pathway Analysis) detected 8 genes which are more abundant in pMSCs than in iwatMSCs, and which are organized in an adipocyte-specific network. These genes include the transcription factor Isl1 (insulin gene enhancer binding protein 1) which was shown to be markedly but transiently upregulated in 3T3-L1 cells shortly after the initiation of differentiation. Its overexpression during early differentiation suppressed Pparg expression and inhibited adipogenesis [26]. Tcf4 (transcription factor 4) is involved in Wnt signaling and forms a complex with $\beta$-catenin [27]. Interestingly, treatment of cardiac stem cells with high glucose concentrations inhibited the $\beta$-catenin/TCF4 pathway and promoted adipogenesis [28]. Therefore, it is conceivable that hyperglycaemia of NZO mice inhibits the expression of Tcf4 in pMSCs, which could ultimately induce their differentiation to adipocytes. Transcripts of two fibroblast growth factors, Fgf1 and Fgf2, are higher expressed in pMSCs than in iwatMSCs and both are involved in the regulation of fat cell differentiation. FGF1 promotes adipogenesis in preadipocytes by increasing the expression of Pparg [29]. In contrast, FGF2 rather inhibits adipogenesis because treatment of proliferating preadipocytes with FGF1 reduced the $F g f 2$ expression markedly, leading to decreased proliferation and increased expression of adipogenic marker genes [30]. Thus, the fact that both $F g f 1$ and $F g f 2$ are expressed at higher levels in pMSCs might explain why their adipogenic cell fate is lower than that of iwatMSCs.

miRNAs have emerged as key epigenetic players during MSC differentiation [22]. They are potent regulators because each miRNA controls and finetunes the expression of several mRNA species. According to the expression profile of miRNAs and the respective target prediction, our data indicate that approximately $40 \%$ of differences in mRNA expression of pMSCs and iwatMSCs are mediated by miRNAs. The fact that two genes, Jade1 and Ubr4, were predicted as targets for the iwatMSC-specific miR-204-5p and the pMSC-specific miR-335-5p, caught our attention. In both cases, two different transcripts were supposed to be affected by the miRNAs. As the resulting protein sequences did not (JADE1) or only moderately (UBR4) differ, their levels and function should be similar in both MSC types.

Interestingly, among the differentially regulated miRNAs, several candidates are known to be involved in adipogenesis, like miR-27-3p, for which we identified 74 targets. 
These include transcription factors like Arnt2 (aryl hydrocarbon receptor nuclear translocator 2) and Nr4a2 (nuclear receptor subfamily 4 group A member 2) and the fatty acid transporter Slc27a1 (also designated FATP1). Interestingly, miR-143-3p, which was the first reported miRNA to be involved in adipogenesis [31], exhibits higher expression in pMSCs and targets Igfbp5 (insulin-like growth factor-binding protein 5) and Hmgcr (HMG-CoA reductase involved in cholesterol synthesis).

Among the differentially expressed miRNAs is also miR-31-5p, which we identified in an obesity locus on chromosome 4and to affect expression of genes relevant for glucose transport and insulin signaling [32]. miR-31-5p was shown to be upregulated by BMP2 in MSCs and to inhibit adipogenesis [33]. Interestingly, the miRNA-335-5p, highly abundant in pMSCs, regulates, among other genes, Cav1 (caveolin 1). As inhibition of Cav1 impairs adipogenesis of bone marrow-derived MSCs [34], it can be hypothesized that adipocyte differentiation of pMSCs, beside other mechanisms, is inhibited via miR-335-Cav1 interaction

Furthermore, also several of the 58 regulated miRNAs were previously associated with pro-fibrotic events. For instance, anti-fibrotic miRNAs miR-221-3p [35], miR-196a$5 p$ [36], and miR-20a-3p [37] were lower expressed in pMSCs and higher in iwatMSCs, while pro-fibrotic miRNAs miR-10a-5p [38] and miR-23b-3p [39] were enriched in pMSCs. More specifically, miR-10a-5p overexpression was shown to promote COL1A1, COL1A3, $\alpha$-SMA, and TGF- $\beta 1$ protein expression and thus increased the levels of atrial fibrillation and cardiac fibroblasts in rat models [38]. It is known that miR-23b-3p together with miR$27 b-3 p$ promotes atrial fibrosis by targeting TGFBR3 [39] and that sponging of miR-196a-5p increases the expression of the lncRNA H19 which leads to an increased fibroblast activation through COL1A1 in the human fibroblast cell line MRC-5 [36]. Therefore, the cell-specific miRNA signature might, in part, explain a fibrogenesis-favoring cell fate of pMSCs.

Several internal and external factors influence proliferation and differentiation of MSCs and these factors include exposure to a range of secreted proteins. Insights into the secretome of different MSCs species could contribute to a better understanding of respective signaling functions. We did not directly analyze the secretome of pMSCs and iwatMSCs but performed an in-silico analysis for transcripts encoding for proteins that are released. Generally, our analysis showed a higher number of genes encoding secreted protein in pMSCs (110 mRNAs of secreted proteins) compared to iwatMSCs (44 mRNAs of secreted proteins). Among the 10 secreted proteins that were predicted to be encoded by genes highly expressed in iwatMSCs, Sfrp2 (secreted frizzled related protein 2) is a known modulator of the Wnt signaling pathway and is described to be a mediator of adipogenic and neuronal differentiation in dental tissue-derived MSCs [40]. Mmp2 and Mmp19 (matrix metallopeptidases 2 and 19), abundant in iwatMSCs, have a pivotal role in adipocyte differentiation, which is in accordance with the observed higher adipogenic potential of iwatMSCs [41,42]. Furthermore, Baek et al. reported about an elevated expression of the secreted protein Galectin-3 (LGALS3) in obesity and T2D. The knockdown of Galectin-3 in 3T3-L1 cells resulted in a reduced adipocyte differentiation and an elevated expression of the adipogenesis mediators Pparg and Cebps, implicating its crucial role in adipogenic differentiation [43]. Even though a few of the depicted protein-coding genes are also linked to pro-fibrogenic properties (Lgals3 [44], Thbs2 [45]), these effects seem to be counterregulated by genes with anti-fibrogenic potential (Mmp19 [46], Fst [47]), which is in line with our functional analyses.

The ten secreted proteins that were predicted to be encoded by genes enriched in pMSCs, including Mgp (matrix gla protein) [48], Igfbp4 (insulin-like growth factor binding protein 4) [49], and Igfbp7 [50], depicted candidates which associate with pro-adipogenic events. This mirrors the in-principle capacity of pMSCs to develop into fat cells, as also observed in our experimental data. However, future studies have to be performed to measure the putatively secreted proteins in the supernatant of pMSCs and iwatMSCs. Interestingly, several prominent pro-fibrogenic proteins were predicted to be enriched in pMSCs, such as Col3a1 (collagen type III alpha 1 chain) [42], Cxcl14 (C-X-C motif chemokine 14) [51], Angptl2 [52], Slit3 (slit guidance ligand 3) [53], and Smoc2 (sparc-related modular 
calcium binding protein-2) [54]. One of the leading fibrogenesis markers, COL3A1, which is regulated by TGF- $\beta$, was predicted to be secreted by pMSCs in our secretome analysis [42]. In addition, it is known that ANGTPL2, also regulated by TGF- $\beta$, has an inflammatory and fibrogenic potential [52] and that suppression of SMOC2 ameliorates kidney, pulmonary, and liver fibrosis [54-56].

The limitation of our study is the fact that several parts are based on in-silico analysis which only allows speculations. Thus, future studies are needed to confirm our results and to clarify which factors induce an elevated adipogenesis in pMSCs.

\section{Conclusions}

In conclusion, we show that pMSCs and iwatMSCs markedly differ in their transcriptome and cell lineage commitments. pMSCs displayed a higher fibrogenic, and iwatMSCs a higher adipogenic differentiation capacity in both, control and diabetes-susceptible mice. Thus, elevated pancreas adipogenesis in diabetes-prone animals has to be mediated by other factors, which require further validation in future experiments. Our results may contribute to an improved understanding how pancreatic fat contributes to the development of T2D as discussed recently [57].

\section{Materials and Methods}

\subsection{Animals}

Male C57BL/6J and NZO/HIBomDIfE mice from our own breeding (German Institute of Human Nutrition Potsdam-Rehbruecke, Nuthetal, Germany) were kept at $20 \pm 2{ }^{\circ} \mathrm{C}$ on a 12:12 h light-dark cycle with ad libitum access to drinking water and a standard diet (V1534-300, ssniff, Soest, Germany). At the age of 8 weeks, non-fasted mice were sacrificed by cervical dislocation for preparation of iWAT and pancreas.

All mice were maintained in accordance with the National Institutes of Health guidelines for the care and use of laboratory animals. All treatments were approved by the ethics committee of the State Office of Environment, Health and Consumer Protection (Federal State of Brandenburg, Potsdam, Germany). The study is reported in accordance with the ARRIVE guidelines.

\subsection{Isolation of Mesenchymal Stromal Cells with Adipogenic Potential}

Flow cytometric isolation was performed based on procedures described previously by Quiclet et al. [58], with minor modifications. In brief, minced iWAT or pancreas from C57BL6/J male and NZO/HIBomDIfE were digested at $37^{\circ} \mathrm{C}$ for $1 \mathrm{~h}$ in $2 \mathrm{mg} / \mathrm{mL}$ (iWAT) and $1 \mathrm{mg} / \mathrm{mL}$ (pancreas) of collagenase type II (Worthington Biochemical Corporation, Lakewood, NJ, USA), and samples were centrifuged at $290 \times g$ and $100 \times g$, respectively. Cell suspensions were filtered through $40 \mu \mathrm{m}$ strainers to remove doublets, large debris, and cell aggregates, and washed with sorting media (HBSS $+2 \%$ FBS). Cells were then stained with antibodies against PTPRC (CD45), PECAM (CD31), and LY6A (SCA1) as described before [12]. To achieve accurate sorting, Sca-1+ cells were marked with an $\operatorname{APC}(\lambda \mathrm{e}=561 \mathrm{~nm})$ and CD31+ and CD45+ cells with a FITC fluorophore $(\lambda \mathrm{e}=488 \mathrm{~nm})$. Subsequently, CD45-:CD31-:SCA1+ cells were sorted for expression of SCA1 (positive selection) and CD45/CD31 (negative selection) to segregate mesenchymal stromal cells (MSCs) (Figure 1A). The labelling with fluorescence dyes enabled the segregation of MSCs from other cellular fractions, accounting for $\sim 1 \times 104$ MSCs per mouse and $\sim 15 \%$ of all isolated cells in the pancreas and for $\sim 1 \times 105$ MSCs and $\sim 50 \%$ of all isolated cells in the iWAT (Figure 1B,C). MSCs from the iWAT (iwatMSCs) and pancreas (pMSCs) were sorted using either a FACSAria III Cell Sorter (BD Biosciences, Franklin Lakes, NJ, USA) or a FACSMelody Cell Sorter (BD Biosciences, Franklin Lakes, NJ, USA). Next, pMSCs as well as iwatMSCs from three to five mice were pooled and cultured at an initial seeding density of 30,000 cells per well in 24-well plates. Finally, obtained cells were expanded for 4 days and isolated RNA was used for miRNome and transcriptome analysis of pMSCs and iwatMSCs (Figure 1A,D) as well as for adipogenic or fibrogenic differentiation. 


\subsection{Adipogenic Differentiation and Oil Red O Staining}

Expanded cells were detached and re-seeded for differentiation in 48-well plates at a density of 15,000 cells per well. Adipogenesis was induced with an induction medium (DMEM 4.5\% glucose, 10\% FBS, $1 \%$ penicillin/streptomycin, $5 \mu \mathrm{g} / \mathrm{mL}$ insulin, $125 \mu \mathrm{M}$ indomethacin, $0.5 \mathrm{mM}$ IBMX, $5 \mu \mathrm{M}$ dexamethasone, $1 \mathrm{nM} \mathrm{T3}$ ) for 2 days and then differentiated for seven additional days with a differentiation medium (DMEM $4.5 \%$ glucose, $10 \%$ FBS, $1 \%$ penicillin/streptomycin, $5 \mu \mathrm{g} / \mathrm{mL}$ insulin, $1 \mathrm{nM} \mathrm{T3}, 1 \mu \mathrm{M}$ Rosiglitazone). For quantification of lipid droplets, Oil Red O staining was performed as previously described [5]. Briefly, cells were washed twice with PBS and fixated with $4 \%$ formalin (RotiHistofix, Carl Roth, Karlsruhe, Germany) for $1 \mathrm{~h}$. Oil Red O stock solution was prepared by mixing $0.5 \mathrm{~g}$ Oil Red $\mathrm{O}$ in $100 \mathrm{~mL}$ isopropanol and a working solution by adding $6 \mathrm{~mL}$ of stock solution to $4 \mathrm{~mL}$ of $\mathrm{dH} 2 \mathrm{O}$ and filtering through filter paper (Whatman \#1, Cytiva Life Sciences, Buckinghamshire, UK). Total of $1 \mathrm{~mL}$ of working solution was added per well, and incubated for $1 \mathrm{~h}$ in the dark. Next, working solution was removed and cells were washed multiple times with $\mathrm{dH} 2 \mathrm{O}$ to remove excess stain. Cell staining was analyzed and imaged in an Eclipse Ts1000 microscope (Nikon, Düsseldorf, Germany), plates were dried at RT and Oil Red O was extracted with isopropanol ( $220 \mu \mathrm{L}$ per well). About $100 \mu \mathrm{L}$ of extracted solution was transferred to a clear 96-well plate, absorbance measured at $562 \mathrm{~nm}$ in a Synergy H1 Hybrid Multi-mode Reader spectrophotometer (BioTek/Agilent Technologies, Santa Clara, USA) and calculated using a standard curve.

\subsection{Fibrogenic Differentiation and Immunocytochemistry}

Fibrogenesis of expanded cells $(15,000$ cells per well) was carried out by incubating cells in a fibrogenic medium (DMEM $1 \%$ glucose, $10 \%$ FBS, $1 \%$ penicillin/streptomycin, $1 \mathrm{ng} / \mathrm{mL}$ TGF- $\beta 1$ ) for 5 days.

For immunocytochemistry, cells were fixated with $4 \%$ formalin (Roti-Histofix, Carl Roth, Karlsruhe, Germany) for $1 \mathrm{~h}$. Cells were then permeabilized using Triton X-100 (0.1\% in PBS), blocked with BSA ( $3 \%$ in PBS) and incubated with the primary antibody against aSMA (Sigma-Aldrich \# A5228; St. Louis, Missouri, USA) overnight. Next day, cells were incubated with secondary antibody (Abcam \# ab150116; Berlin, Germany) for $1 \mathrm{~h}$ in dark. Cells were then washed with PBS and incubated with DAPI ( $300 \mathrm{nM})$ for $5 \mathrm{~min}$. Staining was visualized in a BZ900 Fluorescence Microscope (Keyence, Neu-Isenburg, Germany) and analyzed using ImageJ software.

\subsection{RNA Isolation and Real-Time $q P C R$}

RNA of differentiated MSCs was isolated (miRNeasy Micro Kit, Qiagen, Hilden, Germany) and reversed transcribed (M-MVL RT, Promega, Madison, WI, USA) for quantitative real-time PCR. Genes of interest were detected using specific TaqMan (Thermo Fisher Scientific, Waltham, MA, USA) and IDT (Integrated DNA Technologies, Coralville, IA, USA) probes. Expression levels were evaluated using the 2(-Delta CT) method [4] with eukaryotic translation elongator factor 2 (Eef2) as internal control.

For RNA sequencing, isolated MSCs were expanded for 4 days and RNA was isolated according to the manufacturer's protocol (miRNeasy Micro Kit, Qiagen, Hilden, Germany).

\subsection{RNA and Small RNA Sequencing}

Total RNA was mRNA enriched, fragmented, and underwent DNA nanoball synthesis to get sequenced on the BGISeq platform (Yantian District, Shenzhen, China). After transcriptome sequencing, adapters and low-quality reads were filtered and FastQC v0.11.8 was conducted to check quality of the samples. Next, reads were aligned to the reference genome (GRCm38.p6) using STAR v.2.7.0f, and FPKM values for transcripts were determined by STRINGTIE v1.3.6, both with default options for paired reads.

For small RNA sequencing, small RNA enrichment, purification steps, multiple adapter ligation, and amplification steps were performed. Next, unique molecular identifiers (UMI) were synthesized to the first strand and pooling cyclization was performed. The 
final library got sequenced using BGISEQ. BGI delivered all bioinformatic steps including raw data, small RNA identification, classification to miRNA, siRNA and piRNA as well as the determined expression values in TPM (transcripts per million).

\subsection{Bioinformatic Analyses}

Differentially expressed transcript and miRNA analysis were performed using $\mathrm{R}$ (v4.0.2). Transcripts and miRNAs were filtered for mean expression values below 1 FPKM (fragments per kilobase per million) and 1 TPM, respectively. $p$-values were calculated using Welch's $t$-test. For further analysis of the miRNome, only mature miRNA from the smallRNA dataset was used. In this dataset, one outlier sample was discovered and excluded. Volcano plots were generated via the EnhancedVolcano package (v.1.6.0). The RDAVIDWebService package (v1.26.0) was utilized to perform the GO analysis and GOplot (v1.0.2). Additional pathway analyses were performed using Ingenuity Pathway Analysis (IPA, Qiagen Silicon Valley, Redwood City, CA, USA). The R-package ComplexHeatmap (v2.4.3) was used to create heatmaps.

\section{8. miRNA Target Prediction}

The miRNA target gene prediction was performed as previously described [32]. In brief, target genes were determined using three databases (DIANA-TarBase, miRecords, and miRTarBase [59-61]), which list experimentally validated miRNA-mRNA interactions (detected by HITS-CLIP [62], luciferase reporter or in vitro assays).

\subsection{Statistics and Plotting}

As pancreatic stellate cells and pMSCs have common developmental origins and share several similar characteristics in terms of gene expression and functionalities $[63,64]$, we compared the transcriptome of pMSCs with single cell RNA sequencing (scRNAseq) data from mouse pancreatic stellate cells. The comparison revealed a high enrichment of stellate cell marker genes among the top 1000 expressed pMSC genes (odds-ratio:10.9 p-val: 2.2e-16) (Supplementary Table S1).

Statistical significance was analyzed using Student's t test or one-way ANOVA by comparing the test groups with the appropriate control groups. Data are presented as mean values \pm SEM. Statistical significance is expressed as ${ }^{*}<0.05,{ }^{* *}<0.01$, and ${ }^{* * *}<0.001$. Where applicable, the number of replicates $(n)$ was stated in the figure legend. Plots were created using GraphPad 9.0 (GraphPad Software, San Diego, CA, USA).

Supplementary Materials: The following supporting information can be downloaded at: https:/ / www.mdpi.com/article/10.3390/ijms23042108/s1.

Author Contributions: Conceptualization, H.A. and M.S.; methodology, H.A., F.G.-C., G.S.; software, P.G. and M.J.; validation, H.V., T.J.S. and A.S.; formal analysis, H.A., M.S. and G.S.; investigation, H.A., F.G.-C. and G.S.; resources, A.S.; data curation, M.J.; writing-original draft preparation, H.A., M.S., A.S.; writing-review and editing, H.V., T.J.S. and A.S.; visualization, H.A.; supervision, A.S.; project administration, T.J.S. and A.S.; funding acquisition, A.S. All authors have read and agreed to the published version of the manuscript.

Funding: This research was funded by the German Ministry of Education and Research (BMBF: DZD grant 82DZD00302) and the Brandenburg State.

Institutional Review Board Statement: All mice were maintained in accordance with the National Institutes of Health guidelines for the care and use of laboratory animals. All treatments were approved by the ethics committee of the State Office of Environment, Health and Consumer Protection (Federal State of Brandenburg, Potsdam, Germany). The study is reported in accordance with the ARRIVE guidelines.

Informed Consent Statement: Not applicable.

Data Availability Statement: All data used in this manuscript are available upon request. Accession numbers for raw RNA-seq and miRNA data are GEO: GSE182243. 
Acknowledgments: The skillful technical assistance of Josefine Würfel and Julia Vogt is gratefully acknowledged.

Conflicts of Interest: The authors declare no competing interests.

\section{References}

1. Stadion, M.; Schwerbel, K.; Graja, A.; Baumeier, C.; Rodiger, M.; Jonas, W.; Wolfrum, C.; Staiger, H.; Fritsche, A.; Haring, H.U. Increased Ifi202b/IFI16 expression stimulates adipogenesis in mice and humans. Diabetologia 2018, 61, 1167-1179. [CrossRef] [PubMed]

2. Berry, R.; Jeffery, E.; Rodeheffer, M.S. Weighing in on adipocyte precursors. Cell Metab. 2014, 19, 8-20. [CrossRef] [PubMed]

3. Liu, W.; Li, D.; Cao, H.; Li, H.; Wang, Y. Expansion and inflammation of white adipose tissue-Focusing on adipocyte progenitors. Biol. Chem. 2021, 402, 123-132. [CrossRef] [PubMed]

4. Heilbronn, L.; Smith, S.R.; Ravussin, E. Failure of fat cell proliferation, mitochondrial function and fat oxidation results in ectopic fat storage, insulin resistance and type II diabetes mellitus. Int. J. Obes. Relat. Metab. Disord. 2004, 28 (Suppl. S4), S12-S21. [CrossRef] [PubMed]

5. Ambrosi, T.H.; Scialdone, A.; Graja, A.; Gohlke, S.; Jank, A.-M.; Bocian, C.; Woelk, L.; Fan, H.; Logan, D.W.; Schurmann, A.; et al. Adipocyte Accumulation in the Bone Marrow during Obesity and Aging Impairs Stem Cell-Based Hematopoietic and Bone Regeneration. Cell Stem Cell 2017, 20, 771-784. [CrossRef] [PubMed]

6. Trouwborst, I.; Bowser, S.M.; Goossens, G.; Blaak, E.E. Ectopic Fat Accumulation in Distinct Insulin Resistant Phenotypes; Targets for Personalized Nutritional Interventions. Front. Nutr. 2018, 5, 77. [CrossRef] [PubMed]

7. Vishvanath, L.; Gupta, R.K. Contribution of adipogenesis to healthy adipose tissue expansion in obesity. J. Clin. Investig. 2019, 129, 4022-4031. [CrossRef]

8. Wang, M.-Y.; Grayburn, P.; Chen, S.; Ravazzola, M.; Orci, L.; Unger, R.H. Adipogenic capacity and the susceptibility to type 2 diabetes and metabolic syndrome. Proc. Natl. Acad. Sci. USA 2008, 105, 6139-6144. [CrossRef]

9. Bedogni, G.; Gastaldelli, A.; Manco, M.; De Col, A.; Agosti, F.; Tiribelli, C.; Sartorio, A. Relationship between fatty liver and glucose metabolism: A cross-sectional study in 571 obese children. Nutr. Metab. Cardiovasc. Dis. 2012, 22, 120-126. [CrossRef]

10. Utzschneider, K.M.; Kahn, S.E. Review: The role of insulin resistance in nonalcoholic fatty liver disease. J. Clin. Endocrinol. Metab. 2006, 91, 4753-4761. [CrossRef]

11. Wagner, R.; Jaghutriz, B.A.; Gerst, F.; Oquendo, M.B.; Machann, J.; Schick, F.; Löffler, M.W.; Nadalin, S.; Fend, F.; Königsrainer, A.; et al. Pancreatic Steatosis Associates with Impaired Insulin Secretion in Genetically Predisposed Individuals. J. Clin. Endocrinol. Metab. 2020, 105, 3518-3525. [CrossRef] [PubMed]

12. Quiclet, C.; Dittberner, N.; Gässler, A.; Stadion, M.; Gerst, F.; Helms, A.; Baumeier, C.; Schulz, T.J.; Schürmann, A. Pancreatic adipocytes mediate hypersecretion of insulin in diabetes-susceptible mice. Metabolism 2019, 97, 9-17. [CrossRef] [PubMed]

13. Uhlen, M.; Karlsson, M.J.; Hober, A.; Svensson, A.S.; Scheffel, J.; Kotol, D.; Zhong, W.; Tebani, A.; Strandberg, L.; Edfors, F.; et al. The human secretome. Sci. Signal. 2019, 12, eaaz0274. [CrossRef] [PubMed]

14. Li, X.; Liu, L.; Li, R.; Wu, A.; Lu, J.; Wu, Q.; Jia, J.; Zhao, M.; Song, H. Hepatic loss of Lissencephaly 1 (Lis1) induces fatty liver and accelerates liver tumorigenesis in mice. J. Biol. Chem. 2018, 293, 5160-5171. [CrossRef]

15. Kullander, K.; Klein, R. Mechanisms and functions of Eph and ephrin signalling. Nat. Rev. Mol. Cell. Biol. 2002, 3, 475-486. [CrossRef]

16. Huna, A.; Griveau, A.; Vindrieux, D.; Jaber, S.; Flaman, J.-M.; Goehrig, D.; Azzi, L.; Médard, J.-J.; Djebali, S.; Hernandez-Vargas, H.; et al. PLA2R1 promotes DNA damage and inhibits spontaneous tumor formation during aging. Cell Death Dis. 2021, 12, 190. [CrossRef]

17. Parizadeh, S.M.; Jafarzadeh-Esfehani, R.; Fazilatpanah, D.; Hassanian, S.M.; Shahidsales, S.; Khazaei, M.; Parizadeh, S.M.R.; Ghayour-Mobarhan, M.; Ferns, G.A.; Avan, A. The potential therapeutic and prognostic impacts of the c-MET/HGF signaling pathway in colorectal cancer. IUBMB Life 2019, 71, 802-811. [CrossRef]

18. Wang, W.; Xiao, Z.-D.; Li, X.; Aziz, K.E.; Gan, B.; Johnson, R.L.; Chen, J. AMPK modulates Hippo pathway activity to regulate energy homeostasis. Nat. Cell Biol. 2015, 17, 490-499. [CrossRef]

19. Lopez, P.H.; Aja, S.; Aoki, K.; Seldin, M.M.; Lei, X.; Ronnett, G.V.; Wong, G.W.; Schnaar, R.L. Mice lacking sialyltransferase ST3Gal-II develop late-onset obesity and insulin resistance. Glycobiology 2017, 27, 129-139. [CrossRef]

20. Ekins, T.G.; Mahadevan, V.; Zhang, Y.; D’Amour, J.A.; Akgul, G.; Petros, T.J.; McBain, C.J. Emergence of non-canonical parvalbumin-containing interneurons in hippocampus of a murine model of type I lissencephaly. eLife 2020, 9, e62373. [CrossRef]

21. Tchoukalova, Y.D.; Votruba, S.B.; Tchkonia, T.; Giorgadze, N.; Kirkland, J.L.; Jensen, M.D. Regional differences in cellular mechanisms of adipose tissue gain with overfeeding. Proc. Natl. Acad. Sci. USA 2010, 107, 18226-18231. [CrossRef] [PubMed]

22. Hamam, D.; Ali, D.; Kassem, M.; Aldahmash, A.; Alajez, N.M. microRNAs as regulators of adipogenic differentiation of mesenchymal stem cells. Stem. Cells Dev. 2015, 24, 417-425. [CrossRef] [PubMed]

23. Badimon, L.; Cubedo, J. Adipose tissue depots and inflammation: Effects on plasticity and resident mesenchymal stem cell function. Cardiovasc. Res. 2017, 113, 1064-1073. [CrossRef]

24. Roxburgh, J.; Metcalfe, A.D.; Martin, Y.H. The effect of medium selection on adipose-derived stem cell expansion and differentiation: Implications for application in regenerative medicine. Cytotechnology 2016, 68, 957-967. [CrossRef] [PubMed] 
25. Takahashi, M.; Hori, M.; Ishigamori, R.; Mutoh, M.; Imai, T.; Nakagama, H. Fatty pancreas: A possible risk factor for pancreatic cancer in animals and humans. Cancer Sci. 2018, 109, 3013-3023. [CrossRef]

26. Ma, X.; Yang, P.; Kaplan, W.H.; Lee, B.H.; Wu, L.E.; Yang, J.Y.; Yasunaga, M.; Sato, K.; Chisholm, D.J.; James, D.E. ISL1 regulates peroxisome proliferator-activated receptor gamma activation and early adipogenesis via bone morphogenetic protein 4-dependent and -independent mechanisms. Mol. Cell Biol. 2014, 34, 3607-3617. [CrossRef]

27. Garcia de Herreros, A.; Dunach, M. Intracellular Signals Activated by Canonical Wnt Ligands Independent of GSK3 Inhibition and beta-Catenin Stabilization. Cells 2019, 8, 1148. [CrossRef]

28. Zhang, X.; Meng, K.; Pu, Y.; Wang, C.; Chen, Y.; Wang, L. Hyperglycemia Altered the Fate of Cardiac Stem Cells to Adipogenesis through Inhibiting the beta-Catenin/TCF-4 Pathway. Cell Physiol. Biochem. 2018, 49, 2254-2263. [CrossRef]

29. Newell, F.S.; Su, H.; Tornqvist, H.; Whitehead, J.P.; Prins, J.B.; Hutley, L.J. Characterization of the transcriptional and functional effects of fibroblast growth factor-1 on human preadipocyte differentiation. FASEB J. 2006, 20, 2615-2617. [CrossRef]

30. Hutley, L.J.; Newell, F.S.; Kim, Y.-H.; Luo, X.; Widberg, C.H.; Shurety, W.; Prins, J.B.; Whitehead, J.P. A putative role for endogenous FGF-2 in FGF-1 mediated differentiation of human preadipocytes. Mol. Cell Endocrinol. 2011, 339, 165-171. [CrossRef]

31. Esau, C.; Kang, X.; Peralta, E.; Hanson, E.; Marcusson, E.G.; Ravichandran, L.V.; Sun, Y.; Koo, S.; Perera, R.J.; Jain, R.; et al. MicroRNA-143 regulates adipocyte differentiation. J. Biol. Chem. 2004, 279, 52361-52365. [CrossRef] [PubMed]

32. Gottmann, P.; Ouni, M.; Saussenthaler, S.; Roos, J.; Stirm, L.; Jähnert, M.; Kamitz, A.; Hallahan, N.; Jonas, W.; Fritsche, A.; et al. A computational biology approach of a genome-wide screen connected miRNAs to obesity and type 2 diabetes. Mol. Metab. 2018, 11, 145-159. [CrossRef]

33. Sun, F.; Wang, J.; Pan, Q.; Yu, Y.; Zhang, Y.; Wan, Y.; Wang, J.; Li, X.; Hong, A. Characterization of function and regulation of miR-24-1 and miR-31. Biochem. Biophys. Res. Commun. 2009, 380, 660-665. [CrossRef] [PubMed]

34. Xiang, S.; Li, Z.; Fritch, M.R.; Li, L.; Velankar, S.; Liu, Y.; Sohn, J.; Baker, N.; Lin, H.; Tuan, R.S. Caveolin-1 mediates soft scaffold-enhanced adipogenesis of human mesenchymal stem cells. Stem. Cell Res. Ther. 2021, 12, 347. [CrossRef] [PubMed]

35. Zhou, Y.; Ng, D.Y.E.; Richards, A.M.; Wang, P. microRNA-221 Inhibits Latent TGF-beta1 Activation through Targeting Thrombospondin-1 to Attenuate Kidney Failure-Induced Cardiac Fibrosis. Mol. Ther. Nucleic. Acids 2020, 22, 803-814. [CrossRef] [PubMed]

36. Lu, Q.; Guo, Z.; Xie, W.; Jin, W.; Zhu, D.; Chen, S.; Ren, T. The lncRNA H19 Mediates Pulmonary Fibrosis by Regulating the miR-196a/COL1A1 Axis. Inflammation 2018, 41, 896-903. [CrossRef]

37. Liu, X.; Guo, B.; Zhang, W.; Ma, B.; Li, Y. MiR-20a-5p overexpression prevented diabetic cardiomyopathy via inhibition of cardiomyocyte apoptosis, hypertrophy, fibrosis, and JNK/NF-kappaB signaling pathway. J. Biochem. 2021, 170, 349-362. [CrossRef]

38. Li, P.F.; He, R.H.; Shi, S.B.; Li, R.; Wang, Q.T.; Rao, G.T.; Yang, B. Modulation of miR-10a-mediated TGF-beta1/Smads signaling affects atrial fibrillation-induced cardiac fibrosis and cardiac fibroblast proliferation. Biosci. Rep. 2019, 39, BSR20181931. [CrossRef]

39. Yang, Z.; Xiao, Z.; Guo, H.; Fang, X.; Liang, J.; Zhu, J.; Yang, J.; Li, H.; Pan, R.; Yuan, S.; et al. Novel role of the clustered miR-23b-3p and miR-27b-3p in enhanced expression of fibrosis-associated genes by targeting TGFBR3 in atrial fibroblasts. J. Cell Mol. Med. 2019, 23, 3246-3256. [CrossRef]

40. Lin, X.; Dong, R.; Diao, S.; Yu, G.; Wang, L.; Li, J.; Fan, Z. SFRP2 enhanced the adipogenic and neuronal differentiation potentials of stem cells from apical papilla. Cell Biol. Int. 2017, 41, 534-543. [CrossRef]

41. Chavey, C.; Mari, B.; Monthouel, M.-N.; Bonnafous, S.; Anglard, P.; Van Obberghen, E.; Tartare-Deckert, S. Matrix metalloproteinases are differentially expressed in adipose tissue during obesity and modulate adipocyte differentiation. J. Biol. Chem. 2003, 278, 11888-11896. [CrossRef] [PubMed]

42. Lawrance, I.C.; Rogler, G.; Bamias, G.; Breynaert, C.; Florholmen, J.; Pellino, G.; Reif, S.; Speca, S.; Latella, G. Cellular and Molecular Mediators of Intestinal Fibrosis. J. Crohn's Colitis 2017, 11, 1491-1503. [CrossRef]

43. Baek, J.H.; Kim, S.J.; Kang, H.G.; Lee, H.W.; Kim, J.H.; Hwang, K.A.; Song, J.; Chun, K.H. Galectin-3 activates PPARgamma and supports white adipose tissue formation and high-fat diet-induced obesity. Endocrinology 2015, 156, 147-156. [CrossRef] [PubMed]

44. Al Attar, A.; Antaramian, A.; Noureddin, M. Review of galectin-3 inhibitors in the treatment of nonalcoholic steatohepatitis. Expert Rev. Clin. Pharmacol. 2021, 14, 457-464. [CrossRef] [PubMed]

45. Chen, W.; Wu, X.; Yan, X.; Xu, A.; Yang, A.; You, H. Multitranscriptome analyses reveal prioritized genes specifically associated with liver fibrosis progression independent of etiology. Am. J. Physiol. Gastrointest. Liver. Physiol. 2019, 316, G744-G754. [CrossRef]

46. Jara, P.; Calyeca, J.; Romero, Y.; Plácido, L.; Yu, G.; Kaminski, N.; Maldonado, V.; Cisneros, J.; Selman, M.; Pardo, A. Matrix metalloproteinase (MMP)-19-deficient fibroblasts display a profibrotic phenotype. Am. J. Physiol. Lung Cell Mol. Physiol. 2015, 308, L511-L522. [CrossRef]

47. Mehta, N.; Zhang, D.; Li, R.; Wang, T.; Gava, A.; Parthasarathy, P.; Gao, B.; Krepinsky, J.C. Caveolin-1 regulation of Sp1 controls production of the antifibrotic protein follistatin in kidney mesangial cells. Cell Commun. Signal. 2019, 17, 37. [CrossRef]

48. Li, C.; Li, J.; He, F.; Li, K.; Li, X.; Zhang, Y. Matrix Gla protein regulates adipogenesis and is serum marker of visceral adiposity. Adipocyte 2020, 9, 68-76. [CrossRef]

49. Maridas, D.E.; DeMambro, V.E.; Le, P.T.; Mohan, S.; Rosen, C.J. IGFBP4 Is Required for Adipogenesis and Influences the Distribution of Adipose Depots. Endocrinology 2017, 158, 3488-3500. [CrossRef] 
50. Hu, Z.; Wu, J.; Qin, L.; Jin, H.; Cao, Y.; Zhao, Y. IGFBP7 downregulation or overexpression effect on bovine preadipocyte differentiation. Anim. Biotechnol. 2021, 32, 21-30. [CrossRef]

51. Wang, S.; Shuai, C.; Gao, S.; Jiang, J.; Luan, J.; Lv, X. Chemokine CXCL14 acts as a potential genetic target for liver fibrosis. Int. Immunopharmacol. 2020, 89, 107067. [CrossRef] [PubMed]

52. Cui, L.; Wei, H.; Li, Z.M.; Dong, X.B.; Wang, P.Y. TGF-beta1 aggravates degenerative nucleus pulposus cells inflammation and fibrosis through the upregulation of angiopoietin-like protein 2 expression. Eur. Rev. Med. Pharmacol. Sci. 2020, 24, 12025-12033. [PubMed]

53. Gong, L.; Wang, S.; Shen, L.; Liu, C.; Shenouda, M.; Li, B.; Liu, X.; Shaw, J.A.; Wineman, A.L.; Yang, Y.; et al. SLIT3 deficiency attenuates pressure overload-induced cardiac fibrosis and remodeling. JCI Insight 2020, 5, e136852. [CrossRef] [PubMed]

54. Gerarduzzi, C.; Kumar, R.K.; Trivedi, P.; Ajay, A.K.; Iyer, A.; Boswell, S.; Hutchinson, J.N.; Waikar, S.S.; Vaidya, V.S. Silencing SMOC2 ameliorates kidney fibrosis by inhibiting fibroblast to myofibroblast transformation. JCI Insight 2017, 2, e90299. [CrossRef] [PubMed]

55. Luo, L.; Wang, C.C.; Song, X.P.; Wang, H.M.; Zhou, H.; Sun, Y.; Wang, X.K.; Hou, S.; Pei, F.Y. Suppression of SMOC2 reduces bleomycin (BLM)-induced pulmonary fibrosis by inhibition of TGF-beta1/SMADs pathway. Biomed. Pharm. 2018, 105, 841-847. [CrossRef] [PubMed]

56. Yun, Y.; Feng, L.; Hao, Q. Secreted modular calcium-binding protein 2 promotes high fat diet (HFD)-induced hepatic steatosis through enhancing lipid deposition, fibrosis and inflammation via targeting TGF-beta1. Biochem. Biophys. Res. Commun. 2019, $509,48-55$.

57. Wagner, R.; Eckstein, S.S.; Yamazaki, H.; Gerst, F.; Machann, J.; Jaghutriz, B.A.; Schürmann, A.; Solimena, M.; Singer, S.; Königsrainer, A.; et al. Metabolic implications of pancreatic fat accumulation. Nat. Rev. Endocrinol. 2022, 18, 43-54. [CrossRef] [PubMed]

58. Schulz, T.; Huang, T.L.; Tran, T.T.; Zhang, H.; Townsend, K.L.; Shadrach, J.L.; Cerletti, M.; McDougall, L.E.; Giorgadze, N.; Tchkonia, T.; et al. Identification of inducible brown adipocyte progenitors residing in skeletal muscle and white fat. Proc. Natl. Acad. Sci. USA 2011, 108, 143-148. [CrossRef]

59. Chou, C.H.; Chang, N.W.; Shrestha, S.; Hsu, S.D.; Lin, Y.L.; Lee, W.H.; Yang, C.D.; Hong, H.C.; Wei, T.Y.; Tu, S.J.; et al. miRTarBase 2016: Updates to the experimentally validated miRNA-target interactions database. Nucleic Acids Res. 2016, 44, D239-D247. [CrossRef]

60. Vlachos, I.S.; Paraskevopoulou, M.D.; Karagkouni, D.; Georgakilas, G.; Vergoulis, T.; Kanellos, I.; Anastasopoulos, I.L.; Maniou, S.; Karathanou, K.; Kalfakakou, D.; et al. DIANA-TarBase v7.0: Indexing more than half a million experimentally supported miRNA:mRNA interactions. Nucleic Acids Res. 2015, 43, D153-D159. [CrossRef]

61. Xiao, F.; Zuo, Z.; Cai, G.; Kang, S.; Gao, X.; Li, T. miRecords: An integrated resource for microRNA-target interactions. Nucleic Acids Res. 2009, 37, D105-D110. [CrossRef] [PubMed]

62. Thomson, D.; Bracken, C.P.; Goodall, G.J. Experimental strategies for microRNA target identification. Nucleic Acids Res. 2011, 39, 6845-6853. [CrossRef] [PubMed]

63. Kordes, C.; Sawitza, I.; Götze, S.; Häussinger, D. Stellate cells from rat pancreas are stem cells and can contribute to liver regeneration. PLoS ONE 2012, 7, e51878. [CrossRef] [PubMed]

64. Kordes, C.; Sawitza, I.; Gotze, S.; Haussinger, D. Hepatic stellate cells support hematopoiesis and are liver-resident mesenchymal stem cells. Cell Physiol. Biochem. 2013, 31, 290-304. [CrossRef] 